\title{
Apoptosis induced by the fungal pathogen gliotoxin requires a triple phosphorylation of Bim by JNK
}

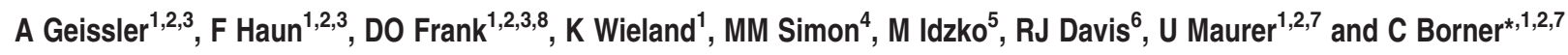

We previously reported that gliotoxin (GT), the major virulence factor of the mold Aspergillus fumigatus causing invasive aspergillosis $(\mathrm{IA})$ in immunocompromised patients, induces apoptosis in a Bak-dependent manner. The signaling pathway leading to Bak activation and subsequent mitochondrial outer membrane permeabilization (MOMP) is elusive. Here, we show that GT and the supernatant of $A$. fumigatus (but not its GT-defective mutant) activate the JNK pathway and require a co-operative

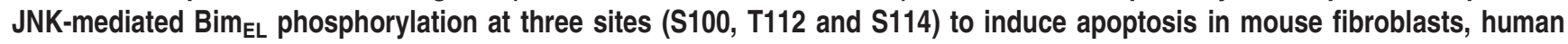
bronchial and mouse alveolar epithelial cells. Cells (i) treated with the JNK inhibitor SP600125, (ii) deleted or knocked down for JNK1/2 or Bim or (iii) carrying the Bim $\mathrm{EL}_{\mathrm{L}}$ triple phosphomutant $\mathrm{S} 100 \mathrm{~A} / \mathrm{T} 112 \mathrm{~A} / \mathrm{S} 114 \mathrm{~A}$ instead of wild-type Bim $\mathrm{EL}$ are similarly resistant to GT-induced apoptosis. Triple-phosphorylated $\mathrm{Bim}_{\mathrm{EL}}$ is more stable, redistributes from a cytoskeletal to a membrane fraction, better interacts with $\mathrm{Bcl}-2$ and $\mathrm{Bcl}-\mathrm{x}_{\mathrm{L}}$ and more effectively activates Bak than the unphosphorylated mutant. These data indicate that JNK-mediated $\mathrm{Bim}_{\mathrm{EL}}$ phosphorylation at S100, T112 and S114 constitutes a novel regulatory mechanism to activate Bim in response to apoptotic stimuli.

Cell Death and Differentiation (2013) 20, 1317-1329; doi:10.1038/cdd.2013.78; published online 5 July 2013

Invasive aspergillosis (IA) is a human opportunistic disease caused by the mold Aspergillus fumigatus, which is highly abundant in the air, soil or on food. ${ }^{1}$ Usually, the intraepithelial immune system of the lung takes care of the inhaled conidia of A. fumigatus. However, in immunocompromised patients, A. fumigatus conidia attach to the lung epithelium, transform into hyphen and invade the lung. ${ }^{2}$ Extensive growth in the lung can cause up to $90 \%$ mortality rates because of antifungal treatment resistance. ${ }^{3}$ For the development of novel therapies, it is therefore crucial to elucidate the molecular mechanism(s) employed by the mold to breach the epithelial lung cell barrier.

Gliotoxin (GT) is the major virulence factor of $A$. fumigatus. ${ }^{4}$ It is secreted in the hyphen state of the fungus and therefore crucial for lung invasion. GT is an epipolythiodioxopiperazine with a reactive disulfide bridge ${ }^{5}$ that can elicit oxidative stress, inhibit NF $\kappa$ B and induce apoptosis in various cell types. ${ }^{6-9} \mathrm{We}$ recently showed that GT triggers rapid apoptosis in mouse embryo fibroblasts via the mitochondrial pathway involving the pore-forming protein Bak. ${ }^{10}$ Pathophysiological relevance for this finding emerged from the studies with Bak $-/$ - mice, which did not develop IA after immunosuppression and infection with $A$. fumigatus. ${ }^{10}$ We therefore proposed that part of the virulence of GT in IA might be through its proapoptotic action in vivo. However, it has remained elusive by which signaling pathway GT activates Bak.

Pro-apoptotic Bak and Bax are activated by so-called $\mathrm{BH} 3-$ only proteins, which facilitate their oligomerization and increase the permeability of the mitochondrial outer membrane (MOMP) by either directly binding to them, or releasing them from the $\mathrm{Bcl}-2$-like survival factors $\mathrm{Bcl}-2, \mathrm{Bcl}-\mathrm{x}_{\mathrm{L}}, \mathrm{Bcl}-\mathrm{w}$ or $\mathrm{Mcl}-1 .{ }^{11}$ Increased MOMP leads to the release of cytochrome $\mathrm{c}$, which, once present in the cytosol, assembles with Apaf-1 and caspase- 9 to the apoptosome, the platform for activating the key effectors of apoptosis, caspases-3 and $-7 .{ }^{12}$ One of the best-characterized $\mathrm{BH}$-only proteins is $\mathrm{Bim} .{ }^{13}$ It is expressed in three isoforms, $\operatorname{Bim}_{E L}, B i m_{L}$ and Bim $S$, of which Bim $E L$ is the most abundant. ${ }^{14}$ The role and regulation of these isoforms are still unclear. In vitro analysis and studies with knockout mice revealed that Bim is involved in apoptosis induced by growth factor deprivation, extracellular matrix detachment (anoikis), glucocorticoids, ER stress agents, UV radiation, negative selection of thymocytes and activation-induced T-cell death. ${ }^{13}$ Apart from transcriptional upregulation in

\footnotetext{
${ }^{1}$ Institute of Molecular Medicine and Cell Research, Albert Ludwigs University Freiburg, Stefan Meier Strasse 17, D-79104 Freiburg, Germany; ${ }^{2}$ Spemann Graduate School of Biology and Medicine (SGBM), Albert Ludwigs University Freiburg, Albertstrasse 19a, D-79104 Freiburg, Germany; ${ }^{3}$ Faculty of Biology, Albert Ludwigs University Freiburg, Schänzlestrasse 1, D-79104 Freiburg, Germany; ${ }^{4}$ Metschnikoff Laboratory, Max-Planck-Institute of Immunobiology, Stübeweg 51, D-79108 Freiburg, Germany; ${ }^{5}$ Department of Pneumology, University Clinic Freiburg, Killianstrasse 5, D-79106 Freiburg, Germany; ${ }^{6}$ Howard Hughes Medical Institute \& Program in Molecular Medicine, University of Massachusetts Medical School, Worcester, MA 01605, USA and ${ }^{7}$ BIOSS, Centre for Biological Signaling Studies, Hebelstrasse 2 , D-79104 Freiburg, Germany

*Corresponding author: C Borner, Institute of Molecular Medicine and Cell Research, Albert Ludwigs University Freiburg, Stefan Meier Strasse 17, Freiburg D-79104, Germany. Tel: + 49761203 9618; Fax: + 49761203 9620; E-mail: christoph.borner@uniklinik-freiburg.de

${ }^{8}$ Current address: Institute of Medical Microbiology and Hygiene, Albert Ludwigs University Freiburg, Hermann Herder Strasse 11, D-79104 Freiburg, Germany. Keywords: apoptosis; Bim; gliotoxin; JNK; anoikis

Abbreviations: GT, gliotoxin; MEF, mouse embryo fibroblasts; WT, wild-type; KO, knockout; DKO, double knockout; DTT, dithiothreitol; SDS-PAGE, sodium dodecyl sulfate polyacrylamide gel electrophoresis; DMEM, Dulbecco's minimal essential medium; FCS, fetal calf serum; poly-HEMA, poly-2-hydroxyethyal methacrylate; PH, poly-HEMA; UV, ultraviolet; GFP, green fluorescent protein; PBS, phosphate buffered saline; IRES, internal ribosome entry site; EtOH, ethanol; RT-qPCR, reverse transcription quantitative polymerase chain reaction; IP, immunoprecipitation

Received 24.9.12; revised 30.4.13; accepted 27.5.13; Edited by G Melino; published online 05.7.13
} 
apoptotic cells, ${ }^{13,15}$ healthy cells can express Bim on mitochondria where it is sequestered by Bcl-2-like survival factors ${ }^{11,13}$ or anchored to the cytoskeleton via dynein light chain (DLC). ${ }^{16}$ In this case, the pro-apoptotic activation of Bim is regulated by posttranslational phosphorylation. In growing cells, Bim $\mathrm{EL}_{\mathrm{L}}$ is phosphorylated at three amino acids (S55/S65/S73) by extracellular signal-regulated kinase-1/2 (ERK1/2). ${ }^{17,18}$ This primes it for proteasomal degradation and keeps Bim at low levels in healthy cells. The significance of this degradation for hematopoietic homeostasis has, however, recently been questioned. ${ }^{19}$ The second reported Bim phosphorylation occurs in response to UV radiation or the negative selection of thymocytes, ${ }^{20,21}$ and perhaps also in response to TRAIL $^{22}$ and involves the c-Jun $\mathrm{N}$-terminal kinases 1 and 2 (JNK1/2)-mediated phosphorylation of Bim $\mathrm{EL}_{\mathrm{L}}$ at $\mathrm{T} 112$ and $\mathrm{Bim}_{\mathrm{L}}$ at $\mathrm{T} 56$. This phosphorylation appears to release Bim from $\mathrm{DLC1}^{21}$ and to increase its binding affinities to $\mathrm{Bcl}-2$-like survival factors, ${ }^{20}$ therefore endowing it with an increased apoptotic potential to activate Bax and Bak, and

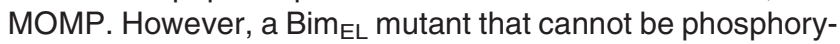
lated at this site (T112A) was only partially able to protect thymocytes from deletion, indicating that other phosphorylation sites of Bim may be crucial for its pro-apoptotic function. 20

Here, we show that the $A$. fumigatus fungal toxin GT induces apoptosis not only in mouse embryonic fibroblasts (MEFs) but also in physiologically more relevant human and mouse lung epithelial cells. This apoptosis involves a rapid, Bax/Bak- and caspase-independent cell detachment and requires the $\mathrm{JNK} 1 / 2$-mediated phosphorylation of $\mathrm{Bim}_{\mathrm{EL}}$ at three sites (S100, T112 and S114). This triple phosphorylation increases the protein stability of $\mathrm{Bim}_{\mathrm{EL}}$, diminishes its sequestration at the cytoskeleton, increases its binding affinity for Bcl-2-like survival factors and activates Bak more effectively.

\section{Results}

GT triggers rapid cell detachment before Bak activation, caspase activation and apoptosis in human lung bronchial epithelial cells. We previously reported that GT induces apoptosis in mouse embryonic fibroblasts in a caspase- and Bak-dependent manner. ${ }^{10}$ Moreover, the fungal toxin triggered rapid cell detachment before Bax/Bak activation. As in vivo GT primarily targets the lung epithelium, we first tested whether the toxin killed BEAS-2B human lung bronchial epithelial cells by the same mechanisms. Indeed, in these cells $1 \mu \mathrm{M}$ GT induced caspase-dependent apoptosis, which was largely delayed by shRNA-mediated downregulation of Bak but not Bax (Figures 1a-c). Again the cells rapidly detached in response to GT, irrespective of the presence or absence of Bak or Bax (Figure $2 \mathrm{a}$ ) or the inhibition of caspases by Q-VD-OPh (QVD) (Figure 2b). Thus, as with fibroblasts, GT first induces cell detachment followed by Bakand caspase-mediated apoptosis in human lung epithelial cells. The toxin therefore initiates apoptosis on the plasma membrane, rather than in the cytoplasm or directly on mitochondria (see Supplementary Information and Supplementary Figure S1), although it is known to be taken up by cells. ${ }^{23,24}$
GT induces several protein kinase signaling pathways, but only the JNK1/2 pathway is crucial for apoptosis induction. To identify the apoptotic signaling from the cell surface to Bak activation on mitochondria, we monitored in BEAS-2B cells the activation of the signaling kinases AKT, MEK and ERK, p38 MAPK and JNK1/2 in response to GT by using phosphospecific antibodies specific for the active forms of each protein kinase. In addition, we studied the implication of two BH3-only proteins, Bad and Bim, whose pro-apoptotic activities were shown to be regulated by these protein kinases. ${ }^{11,13}$ As GT may induce classical anoikis, we also measured these signaling pathways in the poly-HEMA (PH) model system where cells die because they cannot reattach to $\mathrm{PH}$-coated plates after trypsin detachment. ${ }^{25}$

As shown in the Supplementary Information, both $\mathrm{PH}$ and GT triggered the rapid phosphorylation/activation of AKT, MEK and ERK and increased the expression and phosphorylation of Bad at its S112 site. However, none of these events were required for GT-induced apoptosis (Supplementary Figures S2 and S3). We next examined whether GT activated the family of stress-activated protein kinases p38 MAPK and JNK. BEAS-2B cells express the $\mathrm{p} 38 \alpha$ and $\gamma$ isoforms, which can be distinguished by isoform-specific antibodies (Figure 3a and Supplementary Figures S4a, b). Moreover, JNK1 and 2 are readily detected as two bands on SDS-PAGE (Figure 3a). Phosphorylation of both $\mathrm{p} 38 \alpha$ and $\gamma$ as well as JNK1/2 increased within minutes after GT treatment, whereas caspase-3 and its substrate PARP were cleaved only after $2 \mathrm{~h}$ (Figure 3a). As a control, we irradiated the cells with UV, a known stimulus of both the p38 and JNK signaling pathways (Figure $3 a) .{ }^{26}$ To identify whether p38 $\alpha$ and/or $\gamma$ activation were crucial for GT-induced apoptosis, we treated BEAS-2B cells with the pharmacological p38 inhibitors PD169316 and SB203580. Neither inhibitor was capable of delaying GTinduced apoptosis (Figure $3 e$ ). Thus, despite their rapid phosphorylation/activation by GT, the p38 $\alpha$ and $\gamma$ isoforms are not required for GT-induced effector caspase activation and apoptosis in BEAS-2B cells.

We therefore looked at the role of JNK1/2 in GT-induced apoptosis by treating BEAS-2B cells with the JNK inhibitor SP600125. This inhibitor effectively diminished the autophosphorylation of JNK1/2 at T183/Y185, caspase-3 processing and PARP cleavage at $180 \mathrm{~min}$ (Figures $3 \mathrm{a}$ and $5 \mathrm{a}, \mathrm{b}$ ), as well as caspase-3 activity (Figure $3 b$ ) and apoptosis (Figure 3d) during the first $6 \mathrm{~h}$ of GT treatment. As a positive control, the JNK inhibitor also markedly suppressed caspase-3 activity induced by UV (Figure 3b). However, it did not affect PHinduced anoikis (Figure 3b), indicating that GT and anoikis do not mediate apoptosis of BEAS-2B epithelial cells via the same signaling pathways. To make sure that JNK1/2 is the major apoptotic signal transducer of GT, we measured the apoptosis sensitivity in JNK1/2 DKO MEFs. Indeed, when these cells were treated with $1 \mu \mathrm{M}$ GT, they showed an effective blockage of caspase-3 activity (Figure 3c) and processing (Figure $5 \mathrm{~b}$ ) and a marked delay in apoptosis (Figure 3e) as compared with WT MEFs. Moreover, the p38 and MEK inhibitors did not confer additional death protection of JNK DKO MEFs (Figure 3e). Taken together, our results demonstrate that JNK1/2 are the major protein kinase signaling components mediating GT-induced apoptosis. 
a
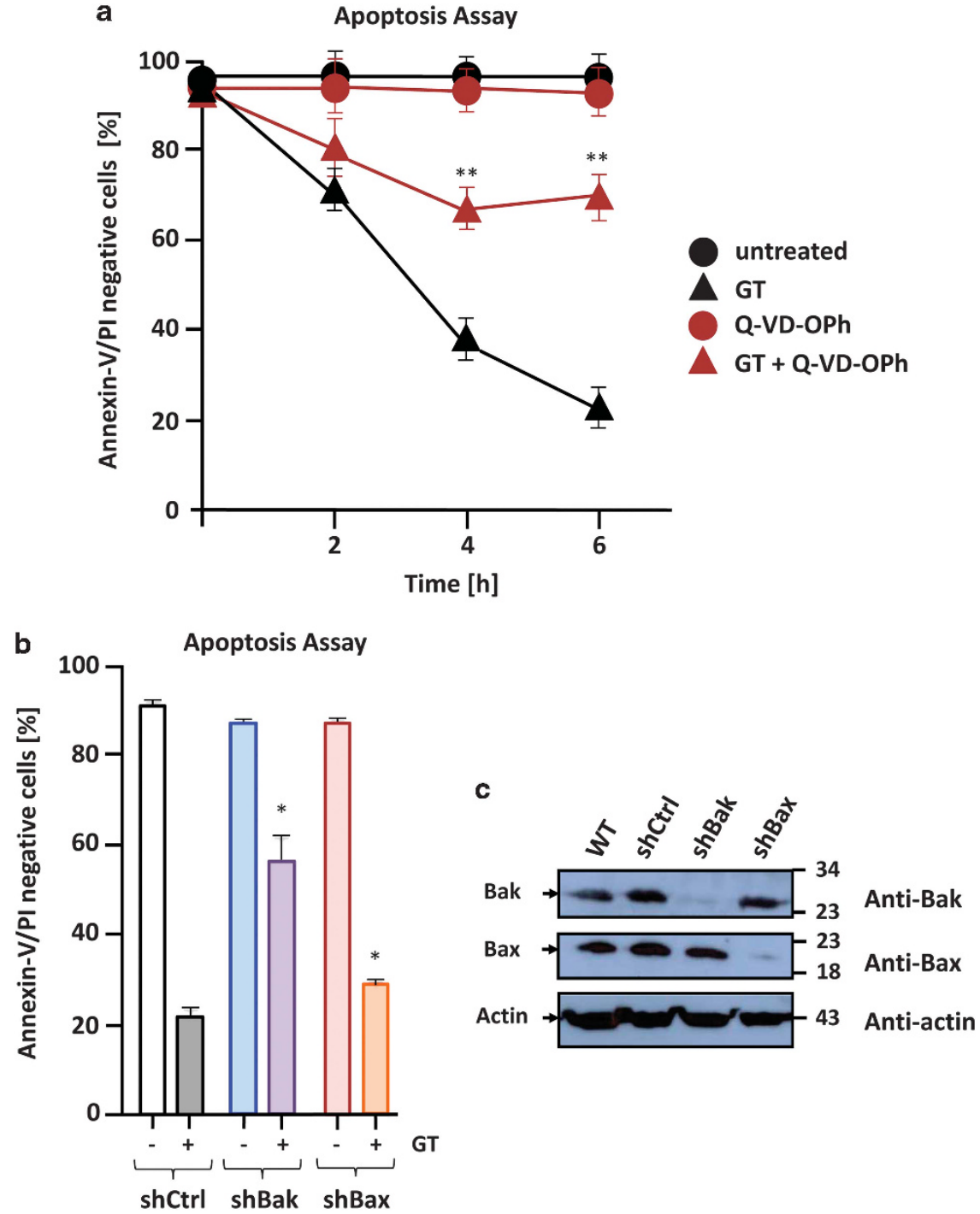

Figure 1 GT induces apoptosis of BEAS-2B cells in a caspase- and majorly Bak-dependent manner. (a) Annexin-V/PI FACS analysis of WT BEAS-2B cells or (b) BEAS$2 \mathrm{~B}$ cells expressing a scrambled control lentiviral shRNA (shCtrl) or shRNAs against Bak (shBak) or Bax (shBax), either untreated $(-)$ or treated $(+)$ with $1 \mu \mathrm{M}$ GT for up to $6 \mathrm{~h}$ in the absence or presence of $25 \mu \mathrm{M}$ of the general caspase inhibitor Q-VD-OPh (a). The graphs show the means of at least three independent experiments \pm S.E.M. $P$-values: ${ }^{* *} 0.001,{ }^{*} 0.01-0.03, n=4$. (c) Anti-Bax and Bak immunoblotting to show the successful downregulation of Bax or Bak by shRNA. Anti-actin immunoblotting served as control for equal protein loading. Molecular weight markers are indicated on the right. As previously shown with MEFs, ${ }^{10} \mathrm{GT}$-induced cell death of BEAS-2B cells is caspasedependent and majorly involves Bak rather than Bax

Bim is crucial for GT-induced apoptosis and a substrate for JNK1/2-mediated phosphorylation at T112. The most probable downstream target of JNK in apoptosis is Bim, as it was previously shown to be phosphorylated and activated by $\mathrm{JNK}^{20,21}$ Indeed, when we treated Bim KO MEFs (Figure 6a) or WT MEFs, effectively silenced for Bim expression by shRNA (Figure $4 \mathrm{a}$ ), with $1 \mu \mathrm{M}$ GT for $6 \mathrm{~h}$ they showed significantly less caspase-3 activation than WT or shRNA control cells (Figure 4b). Moreover, both Bimdeficient cell lines were markedly protected from apoptosis (Figures 4c, d). In contrast, MEFs deficient for other $\mathrm{BH}$ only proteins such as Bad, Bid, Bmf, Bik or Noxa did not exert any delay in GT-induced apoptosis, although PUMA KO cells were slightly protected (Supplementary Figure S5). Thus,
Bim seems to be the major BH3-only mediator of GT-induced apoptosis.

We first considered the possibility of a transcriptional upregulation of Bim by GT, and therefore we quantified the mRNA levels of Bim isoforms by real-time RT-PCR. To our surprise, the total mRNA levels of all splice variants $\left(\right.$ Bim $_{\mathrm{EL}}$, $\mathrm{Bim}_{\mathrm{L}}$ and $\mathrm{Bim}_{\mathrm{S}}$ ) decreased instead of increasing in response to GT (Supplementary Figure S6a). This downregulation was partially due to the activation of the phosphoinositol-3 kinase (PI3K)/AKT signaling by GT (Supplementary Figures S2b and $\mathrm{S} 3 \mathrm{a}$ ) because it was delayed in the presence of the PI3K inhibitor LY294002 (Supplementary Figure S6a). Although the protein levels of $\mathrm{Bim}_{\mathrm{L}}$ and $\mathrm{Bim}_{\mathrm{S}}$ also diminished concomitant with the $\mathrm{mRNA}, \mathrm{Bim}_{\mathrm{EL}}$ protein levels remained constant 
throughout the entire GT treatment period (Supplementary Figure S6b). Cycloheximide ( $\mathrm{CHX}$ ) chase experiments after $2 \mathrm{~h}$ of GT treatment showed that the Bim $\mathrm{EL}_{\mathrm{L}}$ protein levels were stable for the following $6 \mathrm{~h}$ (Supplementary Figure S6c, right panel). This finding indicates that despite transcriptional repression, Bim $_{\mathrm{EL}}$ seems to be post-translationally stabilized in response to GT.

As both JNK1/2 and Bim were required for GT-induced apoptosis, we tested whether Bim $\mathrm{EL}_{\mathrm{L}}$ (the major isoform expressed in BEAS-2B and MEF cells) was phosphorylated by JNK1/2 at T112, which is a known way to increase its proapoptotic potential. ${ }^{20}$ For that purpose, we used a phosphospecific antibody against this site (anti-pT112 Bim) and detected GT-induced T112 phosphorylation of Bim $\mathrm{EL}_{\mathrm{L}}$ in both
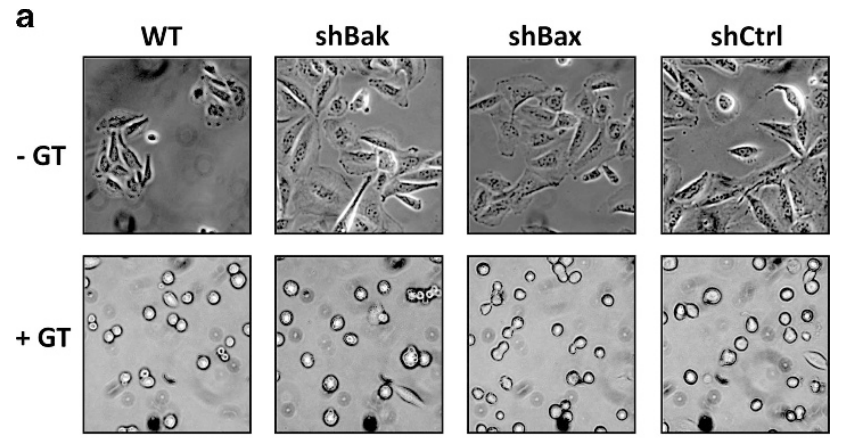

b
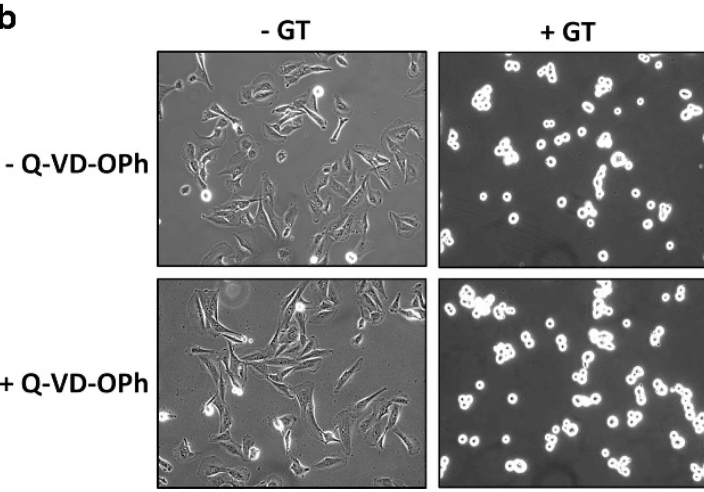

Figure 2 GT-induced cell detachment occurs before Bax or Bak activation. (a) Subconfluent BEAS-2B cells, either WT, carrying a shRNA scrambled construct (shCtrl), or shRNAs against Bak or Bax were left untreated (-GT) or treated with $1 \mu \mathrm{M} \mathrm{GT}(+\mathrm{GT})$ for $1 \mathrm{~h}$ and visualized under the phase contrast light microscope. (b) Microscopic inspection of GT-treated WT cells in the presence or absence of $25 \mu \mathrm{M}$ Q-VD-OPh
BEAS-2B cells (Figure 5a) and MEFs (Figure 5b). This phosphorylation occurred concomitant with JNK1/2 phosphorylation at T183/Y185, and was inhibited by the JNK inhibitor SP600125, confirming that it required JNK activation (Figures $5 \mathrm{a}, \mathrm{b}$ ). To make sure that the T112 $\mathrm{Bim}_{\mathrm{EL}}$ phosphorylation depended on JNK1/2 activation, we performed western blot analysis in WT and JNK1/2 DKO MEFs. Again, concomitant with JNK1/2 activation (pT183/ Y185 detection), GT triggered Bim $\mathrm{EL}_{\mathrm{L}}$ phosphorylation at T112 in WT MEFs (Figure 5b). However, neither T112 phosphorylation nor caspase-3 processing was seen in JNK1/2 DKO cells. Moreover, the JNK1/2 and T112 Bim $\mathrm{EL}_{\mathrm{L}}$ phosphorylations as well as caspase-3 processing were drastically reduced in MEFs treated with the JNK inhibitor (Figure 5b). Interestingly, as described in Supplementary Information, the same apoptotic signaling pathway was activated by the GT treatment of primary murine alveolar epithelial type II cells (Supplementary Figures S7a, b), the main target cells of the $A$. fumigatus mold, as well as in response to the conditioned medium from WT A. fumigatus, but not from a strain lacking GT production (Supplementary Figures S8a-c).

JNK1/2-mediated Bim $\mathrm{EL}_{\text {L }}$ phosphorylation at the T112 site
only partially contributes to GT-induced apoptosis. To address the question whether Bim $_{\mathrm{EL}} \mathrm{T} 112$ phosphorylation by JNK1/2 was required and/or was sufficient for mediating GT-induced apoptosis, we studied the signaling pathway in Bim-deficient primary MEFs that had been reconstituted with WT Bim EL $_{\text {or its phosphodeficient T112A mutant. }}{ }^{20}$ Importantly, both forms of Bim $\mathrm{EL}_{\mathrm{L}}$ were re-expressed at similar levels (Figure 6a). As expected, all cell types showed JNK1/2 phosphorylation/activation after GT stimulation. Moreover, Bim $_{\mathrm{EL}}$ was only phosphorylated at T112 in WT but not in the Bim KO or T112A knock-in mutant cells (Figure 6a). We next measured in all three cell types the extent of GT-induced apoptosis by Annexin-V/PI FACS analysis (Figure 6b) and caspase-3 activity by the DEVDase assay (Figure 6c). Consistent with earlier results, Bim KO MEF cells were significantly protected against GT and showed very little caspase- 3 activity after $6 \mathrm{~h}$. The cells carrying the T112A Bim $_{E L}$ phosphomutant exerted lower caspase-3 activity, and they were partially saved from GT-induced apoptosis at $6 \mathrm{~h}$, but clearly not as much as the Bim KO cells. Thus, surprisingly the JNK1/2-mediated T112 phosphorylation does not much contribute to GT-induced caspase-3 activation and apoptosis.

Figure 3 GT rapidly activates $p 38 \alpha / \gamma$ and JNK1/2, but apoptosis is only inhibited with the JNK inhibitor and in JNK1/2 DKO MEFs. (a) BEAS-2B were left untreated (0) or treated with $1 \mu \mathrm{M}$ GT or $100 \mathrm{~J} / \mathrm{m}^{2} \mathrm{UV}$ light (UV) for 15 to $180 \mathrm{~min}$ and cellular extracts were subjected to immunoblotting using the indicated antibodies. Both stimuli caused phosphorylation of $\mathrm{p} 38 \alpha(\mathrm{pp} 38 \alpha)$ and p38 $\gamma(\mathrm{pp} 38 \gamma)$ at T180 and Y182 and JNK1/2 (pJNK1, pJNK2) at T183 and Y185, although the UV kinetics were faster. Processing of caspase-3 into its active p19/p17 forms and cleavage of its p116 substrate PARP (FL-PARP) into its p85 fragment (CL-PARP) occurred after p38 and JNK phosphorylations. Co-treatment with $20 \mu \mathrm{M}$ of SP600125 (GT + SP) diminished GT-induced JNK1/2 phosphorylation and caspase-3 and PARP processing. Anti-tubulin immunoblotting served as control for equal protein loading. Molecular-weight markers are indicated on the right. (b, c) DEVDase assay of cellular extracts from untreated (Ctrl), GT-treated, UVtreated or PH-cultured BEAS-2B cells (b) or from untreated or GT-treated WT or JNK1/2 DKO MEFs (c) in the absence or presence of SP600125 (SP) or the MEK inhibitor U0126 (UO). Caspase-3/-7 activity was totally blocked in JNK1/2 DKO cells, even more than when treating WT MEFs or BEAS-2B cells with SP600125. The JNK inhibitor also diminished caspase-3 activity in UV-treated, but not in PH-cultured cells. (d, e) Annexin-V/PI staining of BEAS-2B cells (d) or WT or JNK1/2 DKO MEFs (e) exposed to $1 \mu$ M GT for up to $6 \mathrm{~h}$ in the presence or absence of $20 \mu \mathrm{M}$ of either the JNK inhibitor SP600125 (d) or the p38 inhibitors PD169316 or SB203580 (e). In the presence of the JNK inhibitor or when JNK1/2 were deleted, GT-induced apoptosis was drastically delayed, and not further accentuated by the p38 inhibitors. Data in (b-e) are the means of at least three independent experiments \pm S.E.M. $P$-values: ${ }^{* * *}<0.0001,{ }^{* \star} 0.001-0.005, n=4$. NS, not significant 
Two additional JNK-specific phosphorylation sites in Bim $_{\mathrm{EL}}$ cooperate with T112 for GT-induced apoptosis. As Bim KO and JNK1/2 DKO MEFs were more protected against GT-induced apoptosis than the T112A Bim $\mathrm{EL}_{\text {mutant }}$ cells, the $\mathrm{T} 112$ site of $\mathrm{Bim}_{\mathrm{EL}}$ cannot be the only JNK1/2 phoshorylation site important for mediating the killing effect of GT. We therefore screened the amino acid sequence of Bim $_{\mathrm{EL}}$ for putative JNK phosphorylation sites (Ser/Thr residues adjacent to Pro) and found three sites, T38, S100 and S114, which are highly conserved among species (Supplementary Figure S9). S100 and S114 are particularly striking because they are close to the known T112 JNK

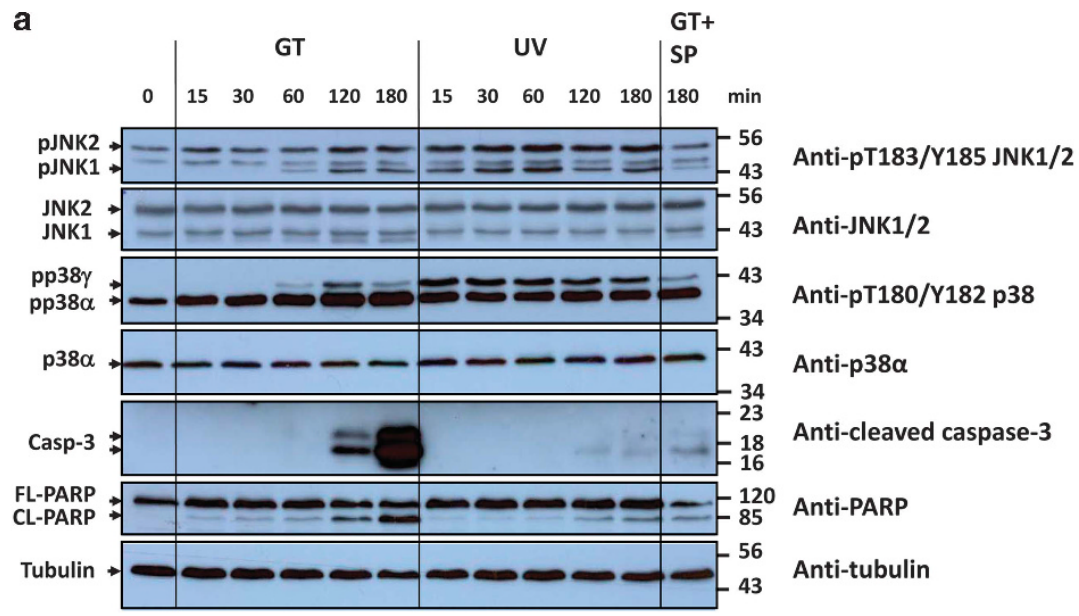

b

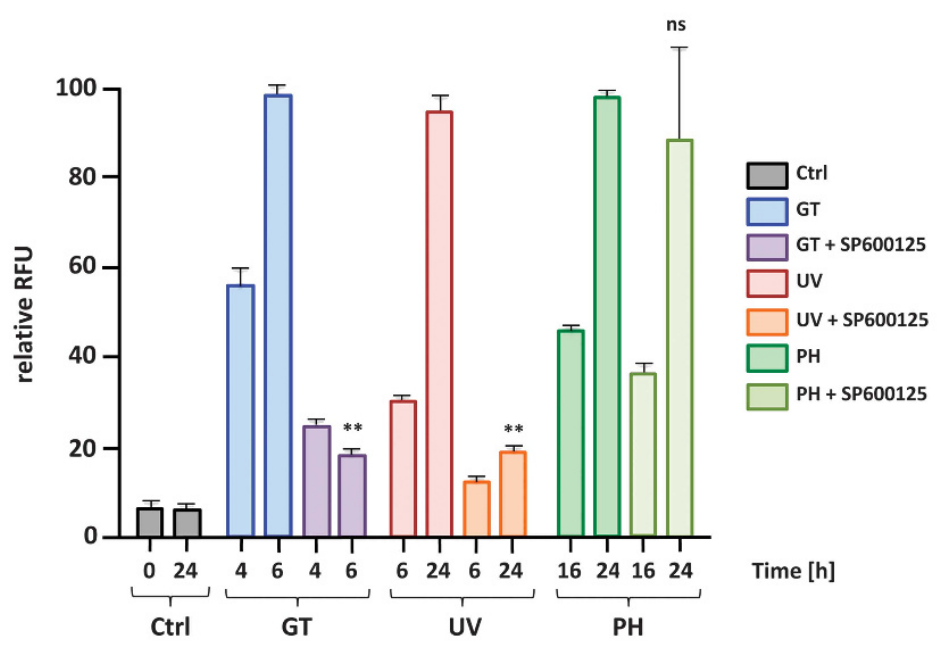

C Caspase-3/-7 (DEVDase) Assay

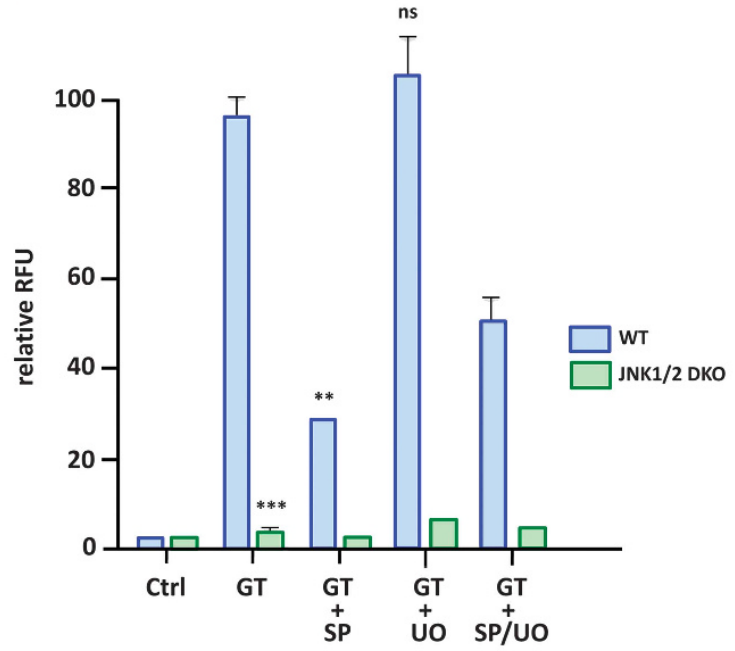

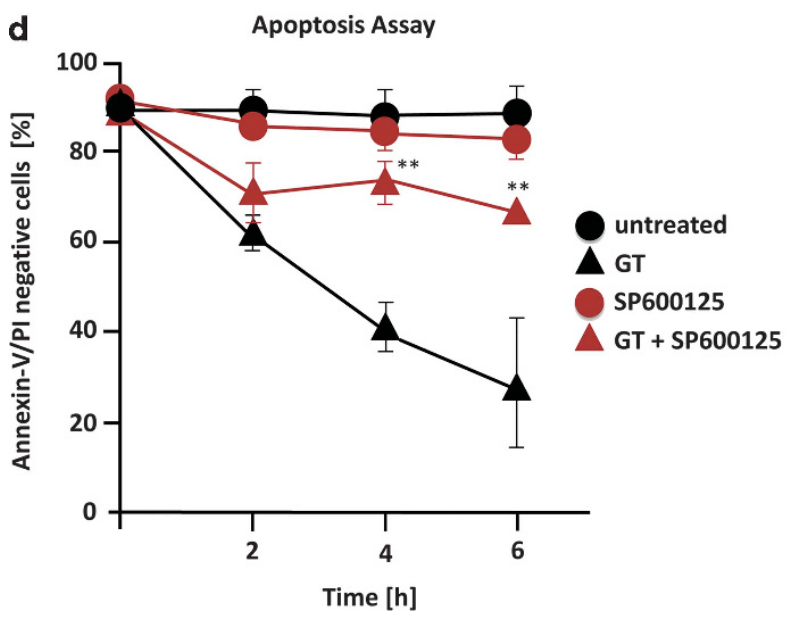

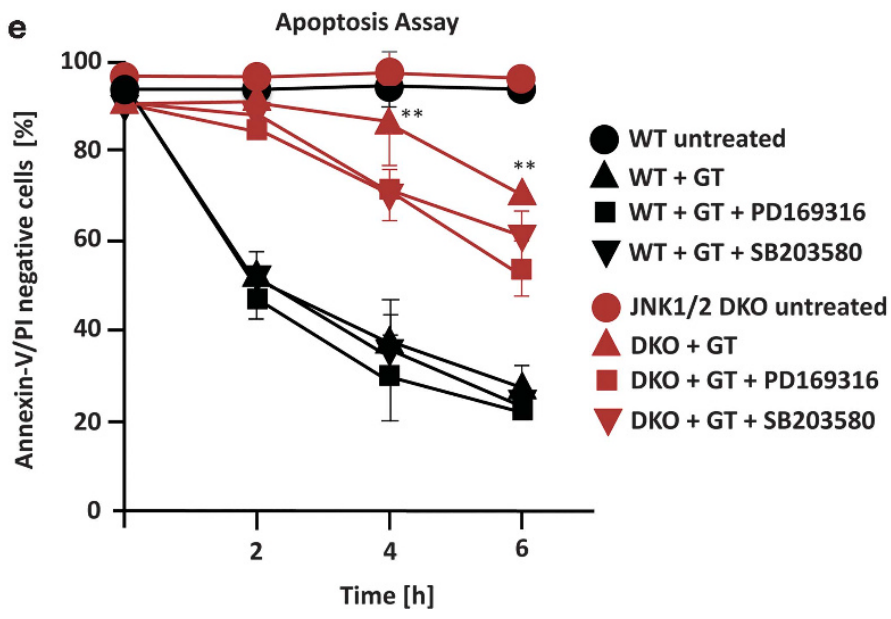



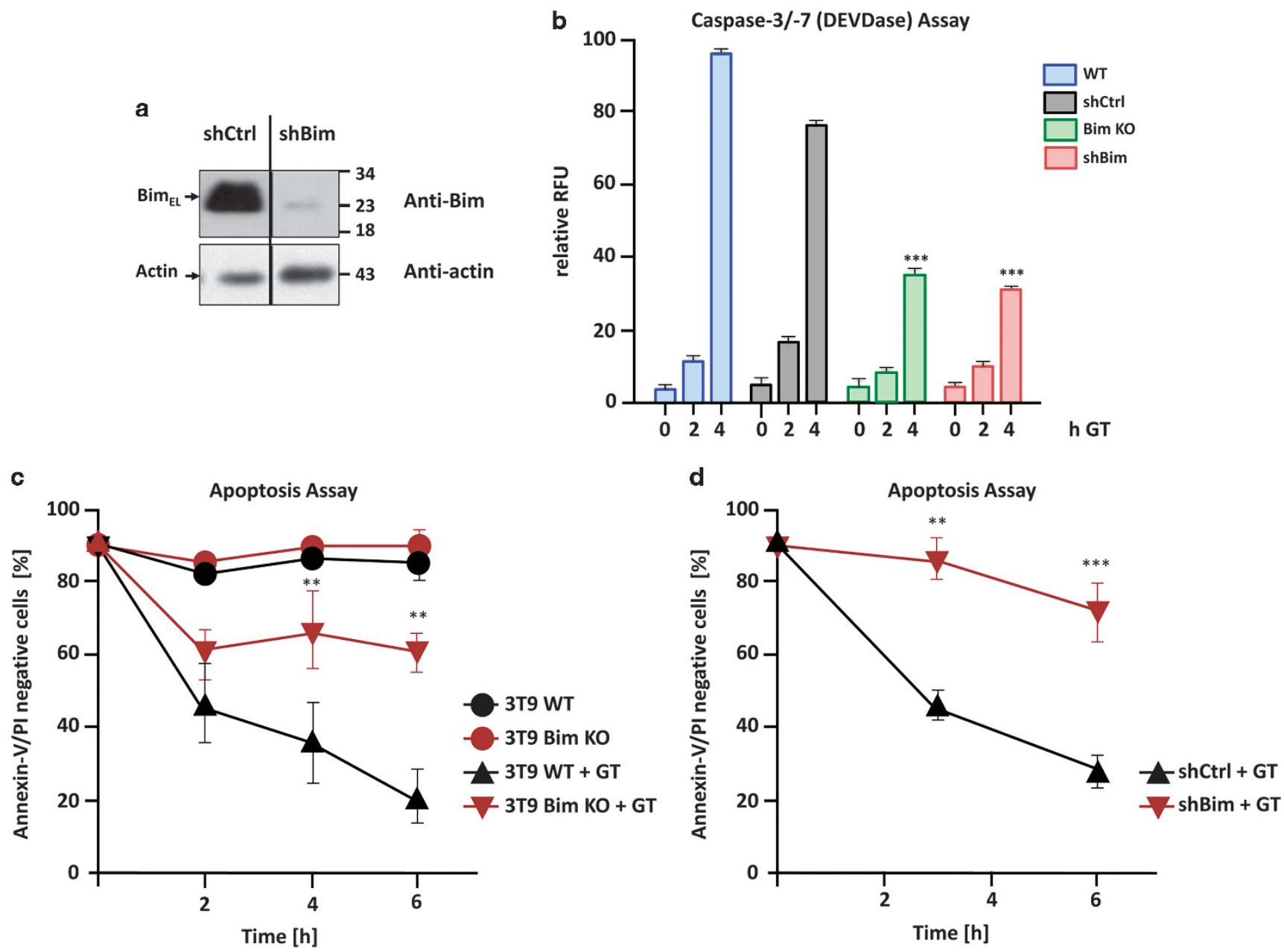

Figure 4 GT-induced caspase-3/-7 activation and apoptosis are suppressed in Bim-deficient MEFs. (a) Efficient downregulation of endogenous Bim $\mathrm{EL}_{\text {b }}$ by shRNA (shBim) as compared with the scrambled control (shCtrl) in MEFs. Actin immunblot as loading control. Molecular-weight markers are indicated on the right. (b) Caspase-3/-7 DEVDase assay or (c, d) Annexin-V/PI FACS analysis of Bim KO MEFs or MEFs expressing a scrambled control shRNA (shCtrl) or an shRNA against Bim (shBim), either untreated or treated with $1 \mu \mathrm{M}$ GT for up to $6 \mathrm{~h}$. Note that caspase-3/-7 activities and the extent of apoptosis were much lower in Bim KO and shBim MEFs than in the WT or scrambled control counterparts at any time of GT treatment. Data are the means of at least three independent experiments \pm S.E.M. $P$-values: ${ }^{* \star *}<0.0001,{ }^{* *} 0.002-0.01, n=4$

phosphorylation site. In addition, the same sites were previously identified in BimL (S44, T56, S58) as in vitro substrates for $\mathrm{JNK}^{21}$ but their physiological significance has not yet been determined. All three sites were mutated to alanines and overexpressed by lentiviral transduction in Bax/ Bak DKO MEFs. In these cells, Bim phosphorylation can be studied without apoptosis induction. As shown in Figure 7a, GT induced a gel shift of WT Bim $\mathrm{EL}_{\text {and the T38A Bim }}$ aL mutant. The band shift was diminished with the S100A and T112A mutants but totally absent with the T112A/S114A double and the S100A/T112A/S114A triple mutants (Figure 7a). These data show that $\mathrm{S} 114$ and/or $\mathrm{S} 100$ of $\mathrm{Bim}_{\mathrm{EL}}$ are novel JNK phosphorylation sites that may be crucial for GT-induced apoptosis. To test the role of each Bim $_{\mathrm{EL}}$ phosphomutant in GT-induced apoptosis, we needed to minimize the cytotoxic potential of Bim overexpression itself. For that purpose, we tried to re-express WT Bim $\mathrm{EL}_{\mathrm{E}}$ and its various phosphomutants in Bim KO MEFs to similar levels as in WT cells. Unfortunately, most of the infected cells died within $24 \mathrm{~h}$ post infection. This was also true for the S100A/

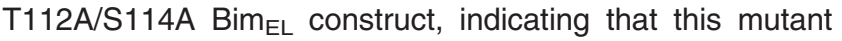
was not defective in apoptosis induction per se. However, we were able to FACS-sort a low number of cells that coexpressed Bim $_{\mathrm{EL}}$ with GFP (IRES), indicating that they tolerated low levels of Bim $\mathrm{EL}_{\mathrm{L}}$. The yield was similar for all Bim $_{\mathrm{EL}}$ mutants (Supplementary Figure S11) and the sorted cells expressed comparable levels of the different Bim $\mathrm{EL}_{\mathrm{L}}$ variants (Figures 7b/c). As in Bax/Bak DKO cells (Figure 7a), GT induced in the reconstituted Bim $\mathrm{KO}$ cells a gel mobility shift of WT Bim $\mathrm{EL}_{\text {and }}$ its S100A, T112A and S114A single mutants, but no such shift was detected with the S110A/ T112A double and the S100A/T112A/S114A triple mutants (Figure 7b). This behavior was also seen on so-called Phostag-SDS gels, which are made with a particular acrylamide (AAL-107) that binds to phosphorylated serines and threonines and produces stepwise gel shifts for each phospho residue in a given protein. Importantly, the gel shift was still evident with the T112A mutant of Bim $\mathrm{EL}_{\mathrm{L}}$ but was lost 

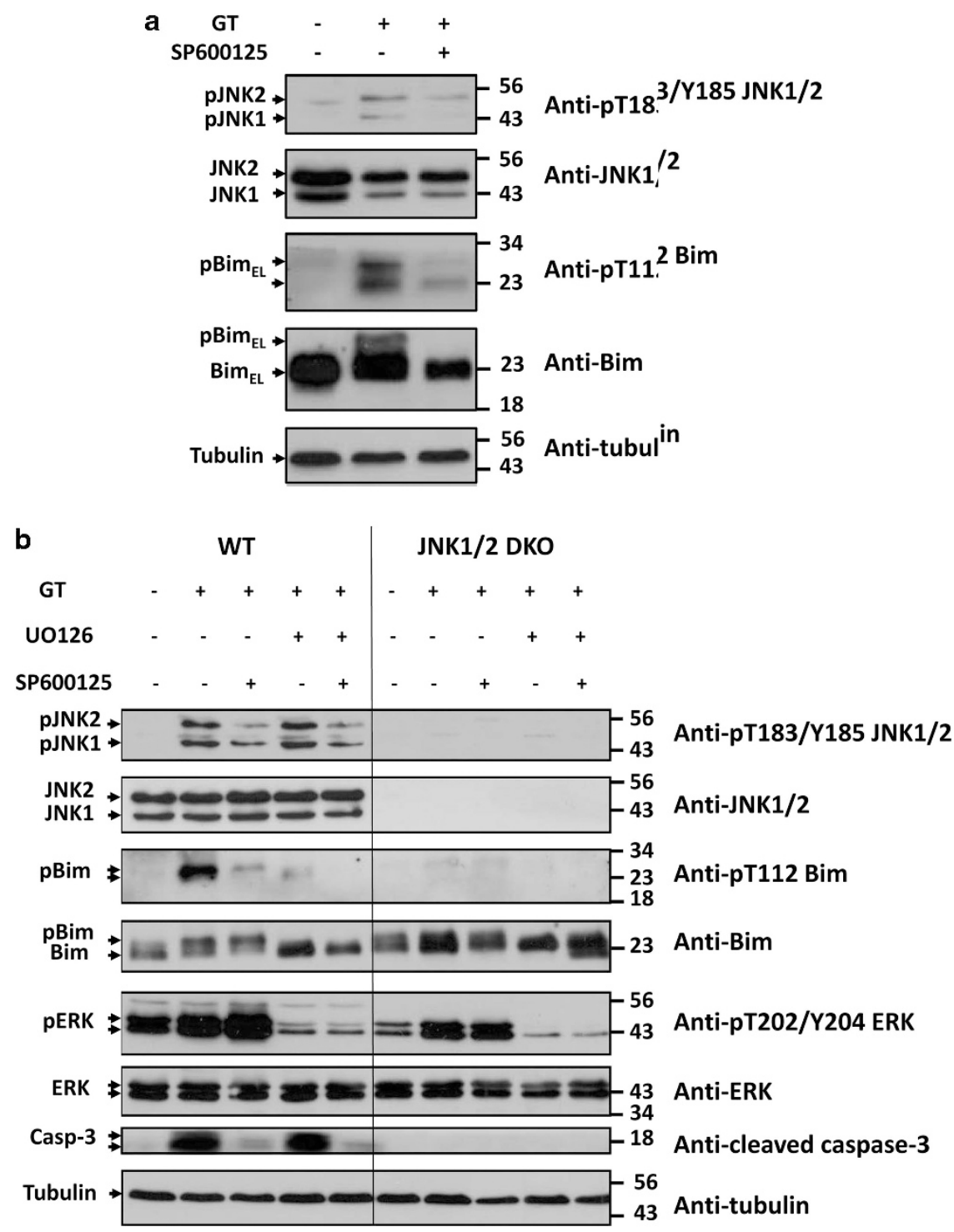

Figure 5 GT induces phosphorylation of Bim $\mathrm{EL}_{\mathrm{L}}$ at T112 in a JNK1/2-dependent manner. (a) Immunoblot analysis of total extracts of BEAS-2B cells or (b) WT and JNK1/2 DKO MEFs, treated or not with $1 \mu \mathrm{M} \mathrm{GT}$ and/or $20 \mu \mathrm{M}$ of the JNK inhibitor SP600125 or the MEK inhibitor U0126 for $6 \mathrm{~h}$. Note that GT induced JNK1/2 phosphorylation at T183 and Y185 and ERK phosphorylation at T202 and Y204 concomitant with Bim EL phosphorylation at T112. Both SP600125 and UO126 diminished the T112 phosphorylation but the phosphorylation was not at all detected in JNK1/2 DKO cells, indicating that MEK/ERK does not directly phosphorylate T112 but may prime this site for JNK $1 / 2$ phosphorylation. The respective antibodies are indicated. Anti-tubulin immunoblot served as control for equal protein loading. Molecular-weight markers are indicated on the right

when this mutant was treated with the JNK inhibitor SP600125 or $\lambda$-phosphatase (Figure 7c). These results clearly show that the GT-induced gel shift of $\mathrm{Bim}_{\mathrm{EL}}$ is primarily due to phosphorylation at S100 and S114 but not at T112.

To determine whether the two additional JNK phosphorylation sites of Bim $\mathrm{EL}_{\mathrm{L}}, \mathrm{S} 100$ and S114, were indeed required for GT-induced apoptosis, we performed quantitative Annexin-VCherry FACS analysis with the GFP-sorted, WT-reconstituted

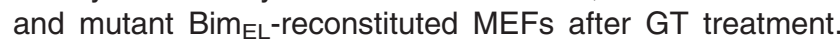
Although Bim KO MEFs re-expressing WT Bim $\mathrm{EL}_{\mathrm{L}}$ or its single T112A or S100A mutants exhibited up to $60 \%$ apoptosis after $8 \mathrm{~h}$ of GT treatment, the cells re-expressing the T112A/S114A double mutant showed only $40 \%$ cell death (Figure $7 d$ ). Strikingly, the cells expressing the S100A/T112A/S114A triple mutant of Bim $\mathrm{EL}_{\mathrm{L}}$ were almost as protected against GTinduced apoptosis as the pMIG vector control Bim KO cells (Figure 7d). Thus, we identified $\mathrm{S} 100$ and S114 as two additional JNK1/2 phosphorylation sites of $\mathrm{Bim}_{\mathrm{EL}}$, which synergize with the T112 phosphosite for effective GT-induced apoptosis.

Triple-phosphorylated Bim $\mathrm{EL}_{\text {is }}$ more stable, associates less with the cytoskeleton, binds better to $\mathrm{Bcl}-2$ and Bcl- $x_{L}$ and activates Bak more efficiently. We finally asked the question by which molecular mechanism the JNK1/2-mediated triple phosphorylation of Bim contributed to GT-induced apoptosis? As shown in Supplementary Figure S6c, the Bim $_{\mathrm{EL}}$ protein seemed to be stabilized after cellular 

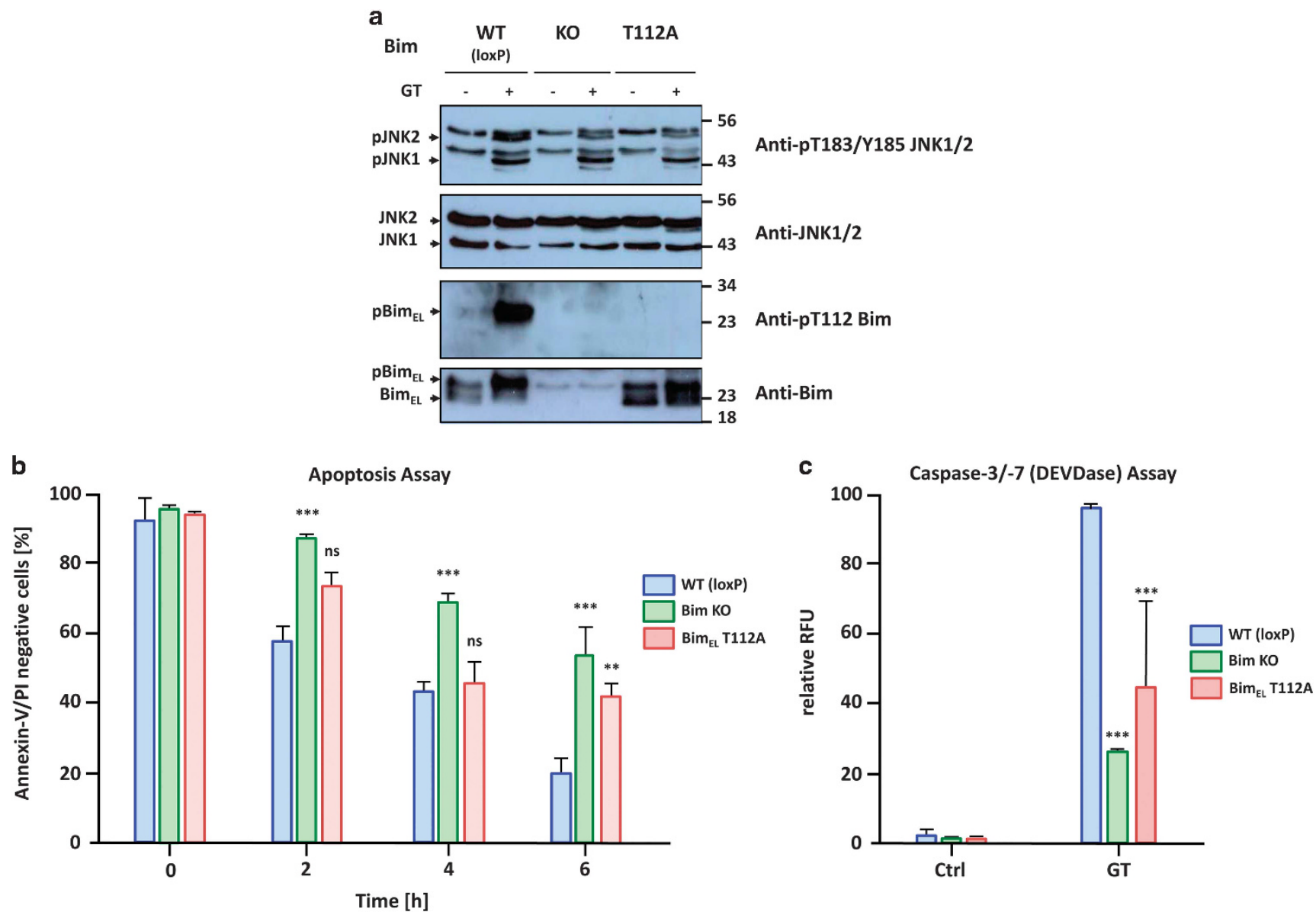

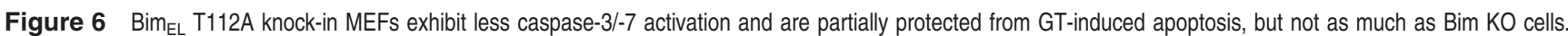
(a) Immunoblot analysis of MEFs derived from WT, Bim KO or Bim KO mice reconstituted with the phosphodeficient T112A Bim EL mutants treated with $1 \mu \mathrm{M} \mathrm{GT} \mathrm{for} 2 \mathrm{~h}$. The respective antibodies are indicated. All cells showed JNK1/2 phosphorylation in response to GT. Bim EL phosphorylation at T112 was only detectable in WT but not in the Bim $\mathrm{KO}$ and T112A mutant cells. Total JNK1/2 immunoblot served a loading control. Total Bim immunoblot showed similar expression levels of Bim $\mathrm{EL}_{\mathrm{EL}}$ A faint cross-reactive band is seen in Bim KO. Molecular-weight markers are indicated on the right. (b) Annexin-V/PI FACS analysis of the four cell lines either untreated (0) or treated with GT for 2-6 $\mathrm{h}$. Bim $\mathrm{KO}$ cells were well protected ( $60 \%$ survival after $6 \mathrm{~h}$ ). MEFs carrying the T112A JNK1/2 phosphomutant also showed a statistically significant protection ( $45 \%$ survival after $6 \mathrm{~h}$ ) but not as much as the Bim KO cells. (c) Caspase-3/-7 DEVDase activity assay after a $6 \mathrm{~h}$ GT treatment. Bim KO cells showed only $30 \%$ caspase-3/-7 activity as compared with WT cells. Caspase-3/-7 inhibition was not as pronounced in Bim $\mathrm{EL}$ T112A cells $(50 \%)$ but statistically significant. The data in (b, c) are the means of at least three independent experiments \pm S.E.M. P-values: ${ }^{* *}<0.0001,{ }^{* *} 0.001-0.005, n=4$. Ns, not significant

treatment with GT because in the presence of $\mathrm{CHX}$ its S100A/T112A/S114A phosphodeficient mutant was more rapidly degraded than the WT form. Moreover, WT, but not

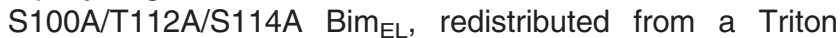
X-100 (TX-100) insoluble cytoskeletal to a TX-100 soluble membrane fraction in response to GT (Supplementary Figures S12a, b), indicating that JNK1/2-mediated triple phosphorylation of Bim EL was required for its detachment from the cytoskeletal DLC1, as previously suggested for Bim $_{L} \cdot{ }^{21}$ Finally, triple-phosphorylated $B_{E L}$ may better bind to Bcl-2-like survival factors and, by consequence, more effectively activate Bak. A higher affinity of $\mathrm{pT} 112 \mathrm{Bim}_{\mathrm{EL}}$ for $\mathrm{Bcl}-2$ has already been reported. ${ }^{20}$ We therefore transiently transfected HEK293 cells with FLAG-tagged versions of WT, $\mathrm{T} 112 \mathrm{~A}$ and S100A/T112A/S114A Bim $\mathrm{EL}_{\text {and }}$ performed antiFLAG IPs of total extracts, followed by anti-Bim, Bcl-2 and $\mathrm{Bcl}-\mathrm{X}_{\mathrm{L}}$ western blotting. As shown in Figure $8 \mathrm{a}$, although all $\mathrm{Bim}_{\mathrm{EL}}$ variants equally pulled down $\mathrm{Bcl}-2$ and $\mathrm{Bcl}-\mathrm{x}_{\mathrm{L}}$ in the absence of GT, there was a higher amount of both survival factors in the anti-FLAG IPs of WT as compared with T112A or triple mutant Bim $\mathrm{EL}_{\mathrm{L}}$ after $4 \mathrm{~h}$ of GT treatment. Importantly, the triple mutant was the weakest binder of $\mathrm{Bcl}-2$ and $\mathrm{Bcl}-\mathrm{x}_{\mathrm{L}}$, indicating that triple phosphorylation of $\mathrm{Bim}_{\mathrm{EL}}$ increased its binding affinity for these survival factors even beyond that already reported for the single T112 phosphorylation. ${ }^{20}$ To determine whether better binding of triple-phosphorylated $B_{E L}$ translated into a more efficient activation of Bak, we performed anti-Bak IPs using a conformation-specific antibody (Ab-1) from extracts of GT-treated HEK293-expressing FLAG-tagged WT, T112A, S114A or triple mutant Bim $\mathrm{EL}_{\text {. }}$ Although Bak was effectively pulled down in the presence of WT Bim $E L$ or its single phosphomutants, only little Bak IP was detected upon expressing the triple mutant of $\mathrm{Bim}_{\mathrm{EL}}$ (Figure 8b). This result shows that GT requires the phosphorylation of Bim at S100, T112 and S114 for efficient Bak activation. 
a

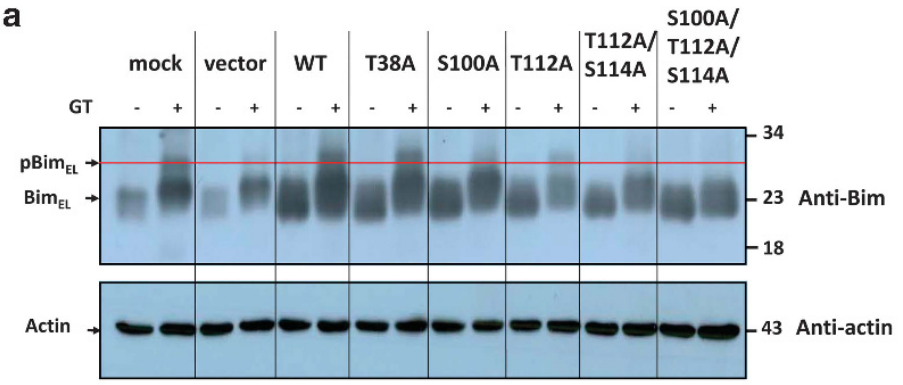

b
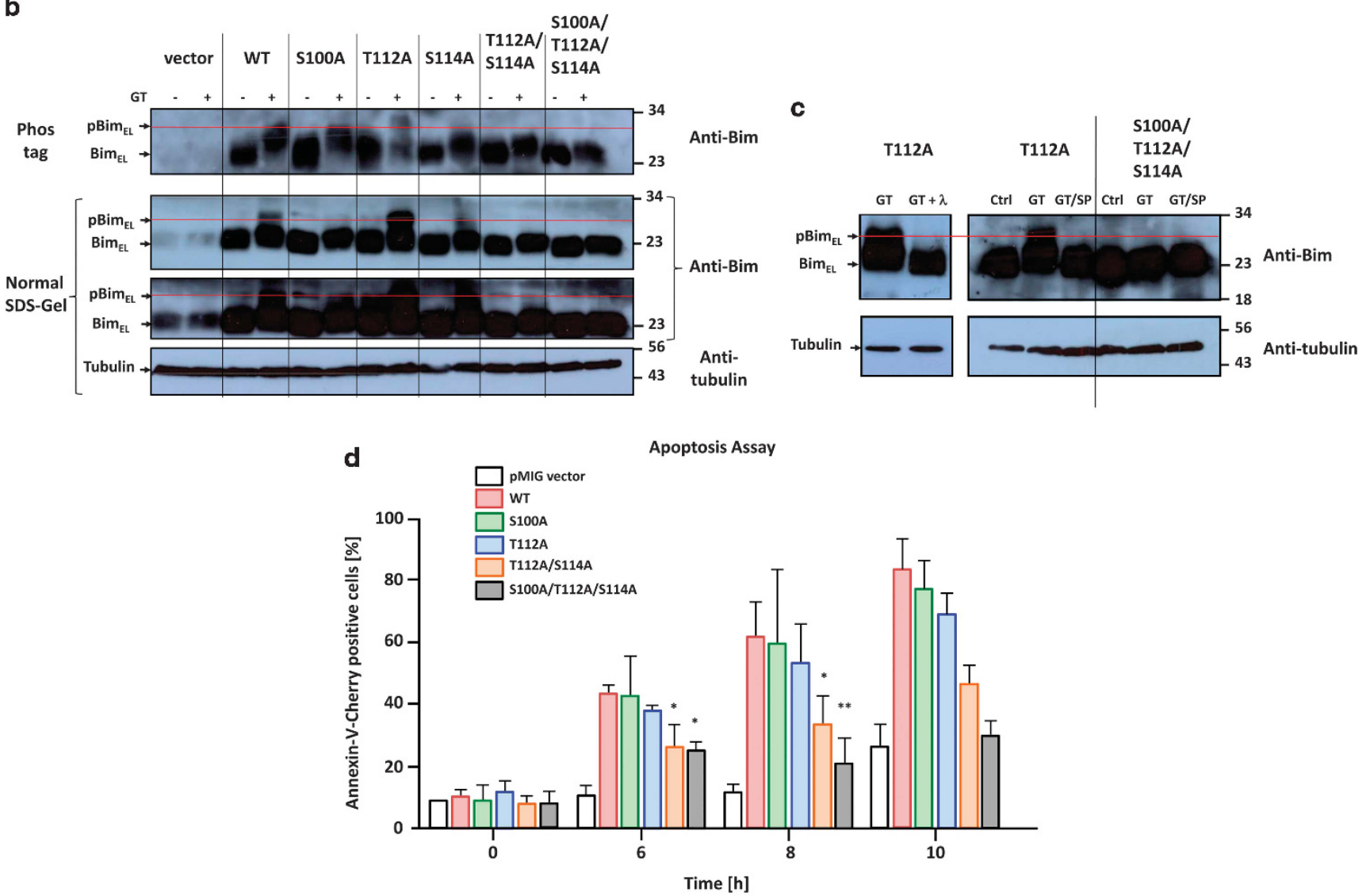

Figure 7 Analysis of GT-induced apoptosis and Bim $\mathrm{EL}_{\mathrm{L}}$ phosphorylation in WT Bim $\mathrm{EL}_{\mathrm{L}}$ and phosphomutant Bim $\mathrm{EL}_{\mathrm{EL}}$ reconstituted Bax/Bak DKO and Bim KO MEFs as well as in HEK293 cells. (a) Anti-Bim immunoblots of total extracts of GT-treated Bax/Bak DKO MEFs or (b) Bim KO MEFs, infected with pMIG-IRES-GFP retroviruses alone (vector)

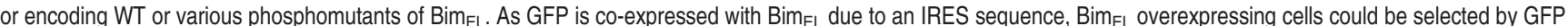
FACS sorting. Re-expressing WT and mutant forms of Bim $\mathrm{EL}_{\text {in }}$ Bim KO (but not Bax/Bak DKO) cells induced spontaneous cytotoxicity. Nevertheless, 5-10\% of the GFPpositive cells could be saved to perform gel-shift analysis (see Supplementary Figure S10). The selected cells contain similar levels of overexpressed WT and mutant Bim $\mathrm{EL}_{\mathrm{L}}$. Mock: uninfected. Both normal and Phostag SDS-gels (see text) are shown. Note that GT induces a gel shift of BimeL (red line) in WT Bim substitution mutants (T38A, S100A, T112A) on both gel types, whereas this shift was entirely lost in the T112A/S114A double and the S100A/T112A/S114A triple Bim $m_{\mathrm{EL}}$ mutants. (c) T112A or triple S100A/T112A/S114A Bim EL mutant-expressing cells were co-treated with GT and the JNK inhibitor SP600125 (SP). In addition, the T112A extract was treated with $\lambda$-phosphatase $(G T+\lambda)$. The mobility shift of T112A Bim $\mathrm{EL}_{\mathrm{L}}$ in response to GT was entirely lost in the presence of the JNK inhibitor or $\lambda$-phosphatase, indicating that it was due to JNK-mediated phosphorylation. No mobility shift was seen for the triple mutant, neither in untreated nor in GT- or GT/SP-treated cases. (d) GFPsorted Bim KO MEFs reconstituted with low amounts of WT Bim $\mathrm{EL}_{\mathrm{L}}$ or its phosphomutants were exposed to $1 \mu \mathrm{M} \mathrm{GT}$ for 6,8 and $10 \mathrm{~h}$ after which apoptosis was quantified by

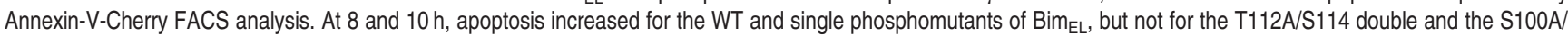
T112A/S114A triple mutants. The data are the means of at least three independent experiments \pm S.E.M. $P$-values: ${ }^{*} 0.004,{ }^{*} 0.02, n=4$. Anti-actin or tubulin immunoblots served as a control for equal protein loading in a-c. Molecular weight markers are indicated on the right

\section{Discussion}

In this study, we used the fungal toxin GT as an apoptotic model agent to uncover a novel signaling pathway that requires $\mathrm{JNK} 1 / 2$-mediated phosphorylation of $\mathrm{Bim}_{\mathrm{EL}}$ at three combined sites (S100, T112 and S114) to trigger Bak- and caspase-dependent apoptosis in fibroblasts (MEFs) as well as kidney (HEK293) and lung epithelial cells (BEAS-2B and primary AEC type II). The signaling pathway appears to be exploited by the fungus $A$. fumigatus to kill lung epithelial cells 


\section{- GT}
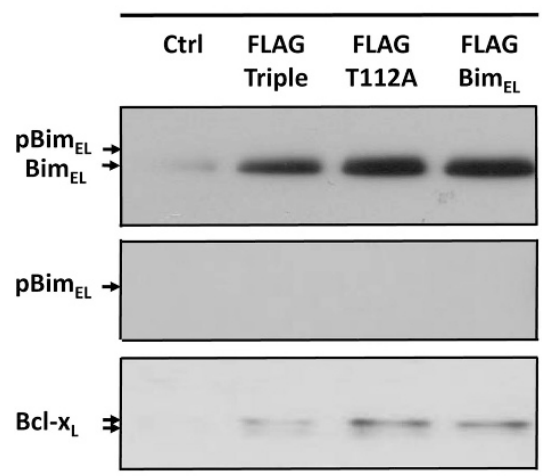

Bcl-2

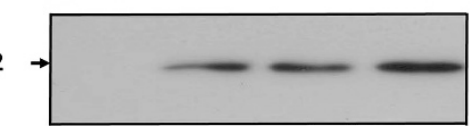

+ GT
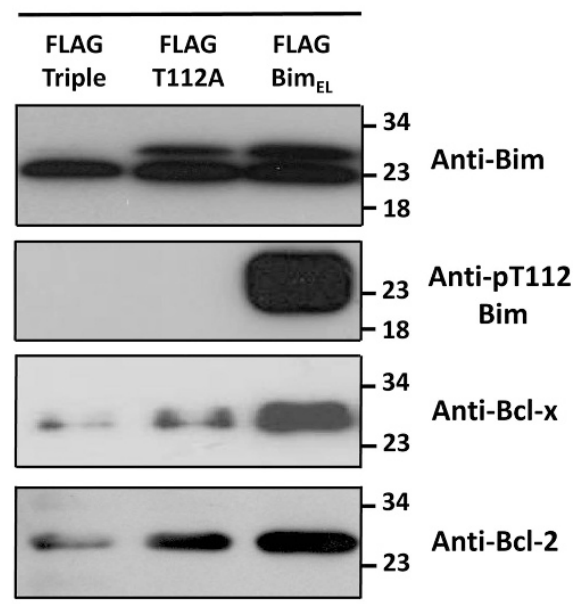

Anti-Bcl-2

b

Anti-Bak Ab-1 IP

Anti-Bak western

$+\mathrm{GT}$

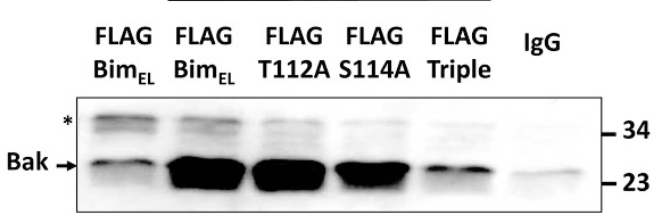

Figure 8 After GT-induced phosphorylation Bim $\mathrm{EL}_{\mathrm{L}}$ interacts better with Bcl-2 or Bcl- $\mathrm{x}_{\mathrm{L}}$ and activates Bak more effectively. (a) Anti-Bim, -pT112 Bim, -Bcl-x and Bcl-2 immunoblots of anti-FLAG IPs/3 $\times$ FLAG-peptide eluates of total CHAPS extracts of HEK293 cells transiently transfected with FLAG-tagged versions of WT Bim EL $_{\text {(FLAG- }}$ $\mathrm{Bim}_{\mathrm{EL}}$ ) or its T112A (FLAG-T112A) or S100A/T112A/S114A triple mutants (FLAG-Triple) and treated with $1 \mu \mathrm{M}$ GT for $4 \mathrm{~h}$. Note that similar amounts of FLAG-Bim $\mathrm{EL}$ and its phosphomutants were immunoprecipitated and all FLAG-Bim $\mathrm{EL}_{\mathrm{L}}$ versions exhibited a similar, basal interaction with Bcl- $\mathrm{x}_{\mathrm{L}}$ and Bcl-2 in the absence of GT (left panels, $-\mathrm{GT}$ ). WT and T112A FLAG-Bim ${ }_{E L}$ showed a gel shift in response to GT that was missing in the triple mutant (right panels, + GT). Only WT FLAG-Bim EL was phosphorylated at T112 and could co-immunoprecipitate more Bcl- $\mathrm{x}_{\mathrm{L}}$ and Bcl-2 than the T112A mutant. The least co-IP was seen with the triple mutant and it was similar to the co-IP in the absence of $\mathrm{GT}$, indicating that GT-induced triple phosphorylation of Bim $\mathrm{EL}_{\mathrm{L}}$ enhanced its binding capacity to $\mathrm{Bcl}-\mathrm{X}_{\mathrm{L}}$ and $\mathrm{BCl}-2$. (b) Anti-Bak immunoblots of anti-Bak Ab-1 immunoprecipitates (using the N-terminal antibody Ab-1, detecting the active conformation of Bak) of the total CHAPS extracts described under (a). In addition, extracts from HEK293 cells transfected with the FLAG-tagged S114A Bim EL phosphomutant were used. Anti-Bak Ab-1 IP is increased in response to GT (compare FLAG- Bim $\mathrm{EL}_{\text {, lanes }}$

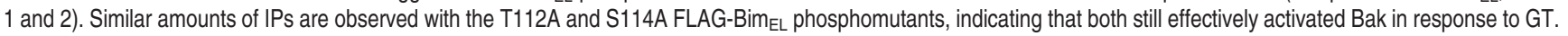
However, the triple mutant did not show any major anti-Bak Ab-1 IP (that is, active Bak). IgG pull down and asterisk ( ${ }^{*}$ ) show some cross-reactive Ig light chains. Molecularweight markers are indicated on the right

in a GT-dependent manner and by this mechanism to eventually infiltrate the lung of IA patients.

As GT induces rapid cell detachment, it most probably acts on the level of the plasma membrane. However, it does not induce classical anoikis. Although both GT and the anoikis inducer poly-HEMA $(\mathrm{PH})$ trigger a rapid, transient activation of AKT, a sustained activation of the p38 MAPK and MEK/ERK pathways and an upregulation and S112 phosphorylation of $\mathrm{Bad}$, none of these events are required for the effector phase of apoptosis. This is not surprising, as both AKT and MEK/ ERK mainly signal for cell survival, ${ }^{27}$ and S112 phosphorylation of Bad has an anti-apoptotic rather than a pro-death effect because it facilitates its binding to the 14-3-3 chaperone, away from the Bcl-2/Bax machinery. ${ }^{28}$ Moreover, although Bim was previously shown to be required for anoikis, ${ }^{29}$ this has been disputed by others. ${ }^{30}$ Why the AKT, ERK and p38 kinase signaling pathways are activated so rapidly by GT remains mysterious. It may either be for another, yet unknown cellular response governed by GT or an attempt of the cell to save itself from apoptosis, which in the end cannot be achieved.
This is supported by our finding that Bim is, at least in part, transcriptionally repressed by the GT-activated PI3K/AKT pathway, although the Bim $\mathrm{EL}_{\mathrm{L}}$ protein is ultimately stabilized and triggers Bak-mediated apoptosis.

In contrast to AKT, ERK and p38 MAPK, we convincingly showed that JNK1/2 are crucial mediators of GT-induced apoptosis but not for $\mathrm{PH}$-induced anoikis (Figure $3 \mathrm{~b}$ ). What might be the mechanism by which GT activates JNK1/2? GT is a hydrophobic molecule, known to enter cells within seconds. ${ }^{23}$ However, in contrast to previous suggestions, ${ }^{23}$ it does not seem to act in the cytoplasm or mitochondria for the following reasons: (i) GT is immediately reduced by high levels of glutathione once entering the cytoplasm, and reduced GT cannot trigger apoptosis (data not shown); (ii) neither reduced nor oxidized GT can induce the cytochrome $c$ release from isolated mitochondria (Supplementary Figure S1). We therefore think that GT initiates apoptosis signaling from the plasma membrane, but different from artificial cell detachment ( $\mathrm{PH}$-induced anoikis), which involves the lack of integrin/focal adhesion kinase (FAK) signaling. ${ }^{25}$ 
Instead, GT may interact with and/or perturb the organization of lipids or proteins within the phospholipid bilayer. Candidate targets in this respect might be the Rho family GTPases RhoA, Rac1 and/or Cdc42, which are known to regulate the link of the actin cytoskeleton to the extracellular matrix and to activate $\mathrm{JNK} 1 / 2$ via kinase phosphorylation/activation of the mixed lineage kinases (MLKs), the Rho kinases (ROCKs) and MAP kinase kinases (MKKs) 4 and 7. ${ }^{31,32}$ A Rac-1 interaction partner POSH was found to trigger JNK-mediated apoptosis and to act as a scaffold protein to organize the upstream kinases in the JNK pathway for effective activation and apoptosis. ${ }^{33}$ It will therefore be interesting to identify the exact molecular target of GT and to uncover how it mediates the apoptotic signal to JNK1/2.

Although the link between GT and JNK activation remains elusive, our data clearly implicate JNK1/2-mediated Bim $\mathrm{EL}$ phoshorylation as the major transducer of GT-induced cytotoxicity. Surprisingly, however, the known T112 phosphorylation of $\mathrm{Bim}_{\mathrm{EL}}$ does not seem to contribute much to caspase-3 activation and apoptosis on its own (Figures 6b, c), and the T112A mutant is still effectively labeled with ${ }^{33} \mathrm{P}$-orthophosphate in cells treated with GT (Supplementary Figure S10). Instead, the JNK1/2-mediated phosphorylation of two additional sites, S100 and S114, cooperatively converts Bim $_{\mathrm{EL}}$ into a highly pro-apoptotic protein, which is released from the cytoskeleton (most probably from DLC1 as reported $^{21}$ ) (Supplementary Figure S12), better interacts with $\mathrm{BCl}-2$ and $\mathrm{Bcl}-\mathrm{x}_{\mathrm{L}}$ (Figure $8 \mathrm{a}$ ) and more effectively activates Bak (Figure 8b). Importantly, the spontaneous killing activity of Bim $\mathrm{EL}_{\mathrm{L}}$ is not affected by this triple phosphorylation as similar cell numbers were obtained after transfecting WT Bim EL or its S100A/T112A/S114A mutant into Bim KO MEFs (Supplementary Figure S11).

Our data extend a previous report by Lei and Davis ${ }^{21}$

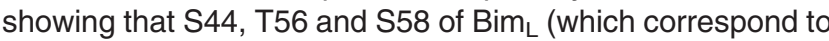

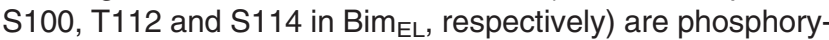
lated in vivo, are substrates of JNK in vitro and lie within the Bim $_{L}$-DLC1 interaction domain. Interestingly, overexpression of active JNK1 and UV radiation provoked a band shift in Bim $L$ that was ablated in a S44A/T56A/S58A triple mutant. Moreover, JNK activation released Bim from the DLC1 complex, but only a phosphomimetic of the T56 site (T56D) (T112 in Bim $_{E L}$ ) was shown to provoke the same release and to slightly increase the pro-apoptotic activity of Bim $\mathrm{L}_{\mathrm{L}}$. The co-operative involvement of all three phosphosites in UV or active JNKmediated apoptosis was, however, not investigated. Here, we show for the first time that not only T112 in Bim $\mathrm{EL}_{\mathrm{L}}$ or T56 in $\mathrm{Bim}_{\mathrm{L}}$ but two additional JNK1/2 phosphorylation sites (S100 and $\mathrm{S} 114$ in $\mathrm{Bim}_{\mathrm{EL}}$ and by analogy $\mathrm{S} 44$ and $\mathrm{S} 58$ in Bim $\mathrm{L}$ ) are required to fully activate $\mathrm{Bim}$ in response to GT and perhaps other apoptotic stimuli. It should be noted that the antiphosphoT112 antibody used in this study most probably binds dual phosphorylated pT112 and pS114 Bim $\mathrm{EL}_{\text {, }}$ as it was raised against a peptide that was phosphorylated at both sites (DKSTQpTPpSPP). ${ }^{20}$ This reinforces our notion that both T112 and S114 are simultaneously phosphorylated by JNK1/2 in response to GT. Further studies are required to elucidate the combined role of S100/T112/S114 phosphorylations of Bim in GT-induced apoptosis in vivo and whether this mechanism is the major cause for IA development in humans.

\section{Materials and Methods}

Reagents. LY294002, PD169316, SB203580, SP600125, U0126 and CHAPS were purchased from Calbiochem (Darmstadt, Germany). Q-VD-OPh was obtained from MP Biomedicals (Eschwege, Germany) and SABOURAUD-2\% glucose agar and MG132 were from Merck-VWR (Darmstadt, Germany). Bovine serum albumin (BSA), Triton X-100, CHX, EZview Red Anti-FLAG M2 Affinity beads and the $3 \times$ FLAG peptide were purchased from Sigma-Aldrich (Taufkirchen, Germany), the $\lambda$-phosphatase was from New England Biolabs (Frankfurt am Main, Germany). Acrylamide and dithiothreitol (DTT) were purchased from Applichem (Darmstadt, Germany). ${ }^{33} \mathrm{P}$-orthophosphate was obtained from GE Healthcare Life Sciences (Freiburg, Germany).

Cells. SV40-transformed BEAS-2B human lung bronchial epithelial cells and HEK293T cells (both ATCC) were grown in RPMI + 10\% FCS. 3T9-immortalized WT, Bim KO, other BH3-only KO and Bax/Bak DKO MEFs as well as primary

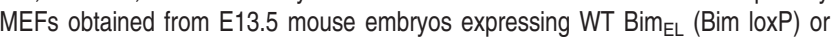
the Bim ${ }_{E L}$ knock-in phosphomutant T112A, or lacking Bim (Bim KO) or both JNK1 and JNK2 (JNK1/2 DKO) expressions were maintained in DMEM + 10\% FCS. Primary alveolar epithelial type II cells (AEC type II) were isolated from C57BL/6 mice as previously described. ${ }^{34}$

Cell fractionation, immunoblotting and antibodies. Cells were lysed on ice for $30 \mathrm{~min}$ in $50-100 \mu \mathrm{l}$ buffer A $(20 \mathrm{mM}$ Tris- $\mathrm{HCl} \mathrm{pH} 7.5,150 \mathrm{mM}$ $\mathrm{NaCl}, 5 \mathrm{mM}$ EDTA pH 8.0, $5 \mathrm{mM}$ Na-pyrophosphate, $1 \mathrm{mM} \mathrm{Na}_{3} \mathrm{VO}_{4}, 20 \mathrm{mM}$ $\mathrm{NaH}_{2} \mathrm{PO}_{4}, \mathrm{pH} 7.6,3 \mathrm{mM} \beta$-glycerophosphate, $10 \mathrm{mM} \mathrm{NaF}, 1 \%$ phosphatase inhibitor cocktail 1 and 2 (Sigma-Aldrich), $1 \times$ protease inhibitor cocktail complete (Roche, Mannheim, Germany), 0.1\% MG-132) containing 1\% Triton-X-100 (TX-100). The whole-cell lysate was briefly spun at 13000 r.p.m., and the supernatant was used for further studies. For separation into TX-soluble and insoluble fractions, the cells were pelleted, resuspended in MSH buffer $(210 \mathrm{mM}$ mannitol, $70 \mathrm{mM}$ sucrose, $20 \mathrm{mM}$ HEPES, pH 7.5, $1 \mathrm{mM}$ EDTA and complete protease inhibitors) and lysed using a syringe with a $23-27 \mathrm{G}$ needle until $50 \%$ of the cells were broken (trypan blue positive). The nuclei were isolated by centrifugation at $500 \times g$ followed by centrifugation at $100000 \times g, 4^{\circ} \mathrm{C}$ for $60 \mathrm{~min}$ to obtain the cytosol and a membrane pellet. The pellet was resuspended in buffer A containing $1 \% \mathrm{TX}-100$. After an additional centrifugation at $100000 \times g, 4^{\circ} \mathrm{C}$ for $60 \mathrm{~min}$, the supernatant contained TX-100-solubilized membrane proteins. The TX-insoluble pellet was resuspended in buffer $\mathrm{A}+1 \%$ SDS and boiled at $95^{\circ} \mathrm{C}$ for $5 \mathrm{~min}$. The protein concentration was determined by the Bradford assay. The supernatant proteins $(20-60 \mu \mathrm{g})$ were separated on SDS-PAGE and transferred to nitrocellulose membranes. The membranes were probed with the rabbit polyclonal anti-Bax $(1: 5000)$ or anti-Bak $(1: 5000$, \#06-536) antibodies from Upstate/ Millipore (Darmstadt, Germany), or the rabbit polyclonals anti-Bad (D24A9), antiBim (C34C5), anti-Bcl- $\mathrm{x}_{\llcorner}$(54H6), anti-ERK1/2 (137F5), anti-JNK1/2, anti-p38\%, anti-PARP, anti-pS112-Bad, anti-pS217/221-MEK, anti-pS473-AKT (193H12), anti-pT180/Y182-p38, anti-pT183/Y185-JNK (98F2), anti-T202/Y204-ERK1/2 or anti-cleaved (Asp175) caspase-3 or the mouse monoclonals anti-p38 $\alpha$, anti-AKT or Bcl-2 (10C4) (all 1:1000) from Cell Signaling Technologies (Frankfurt am Main, Germany). As secondary antibodies, peroxidase-coupled goat anti-mouse, antirabbit or anti-rat IgG were used and the immunodetection was performed using Enhanced Chemiluminescence (Pierce, Rockford, IL, USA). The rabbit polyclonal anti-pT112-Bim antibody was generated and characterized as previously described. ${ }^{20}$ For immunoprecipitations (IPs), the mouse monoclonal anti-Bak antibody AM03 (Ab1, clone TC100, Upstate/Millipore), mainly detecting conformationally changed, active Bak (aa 1-52) was used. As controls of equal loading and organelle purity the mouse monoclonal anti-actin (C4) (1:40000, MP Biomedicals, Clone C4, \#691000), the mouse monoclonal anti-ATPase/OxPhosSubunit V (1:1000, Molecular Probes, \#A21350) or the rat monoclonal anti-tubulin (1:20000, AbD Serotec, clone YL1/2, \#MCA77G) antibodies were used. Phos-tag SDS-PAGE was performed exactly as described by the manufacturer (Wako Chemicals, Neuss, Germany).

Cell death assays. Lyophilized GT was purchased from Sigma-Aldrich. Maximal $60-70 \%$ confluent BEAS-2B and MEFs were treated with $1 \mu \mathrm{M}$ GT for up to $8 \mathrm{~h}$ to induce apoptosis. Alternatively, the cells were irradiated with UV light $\left(100 \mathrm{~J} / \mathrm{m}^{2}\right)$ in a UV Stratalinker (Stratagene, La Jolla, CA, USA). Anoikis was induced in BEAS-2B cells using the poly-2-hydroxyethyal methacrylate (polyHEMA)-coating method. Poly-HEMA (Sigma-Aldrich) is a hydrogel that prevents cells to attach to culture dishes. In brief, $2.4 \mathrm{~g}$ of poly-HEMA was dissolved in $20 \mathrm{ml}$ of $95 \%$ ethanol and shaken at $65^{\circ} \mathrm{C}$ for at least $8 \mathrm{~h}$. A volume of $1.5 \mathrm{ml}$ of a 
1: 10 diluted solution (in EtOH) was added to each well of a six-well plate, and the plates were dried overnight at $37^{\circ} \mathrm{C}$. Apoptosis was quantified by His-GFPAnnexin-V/propidium iodide (PI) or His-CHERRY-Annexin-V FACS analysis as described..$^{35}$ Caspase-3/-7 activity was measured by incubating whole-cell extracts with $60 \mu \mathrm{M}$ fluorogenic DEVD-AMC in $100 \mathrm{mM}$ Hepes- $\mathrm{KOH}$, pH 7.5, containing $10 \mathrm{mM}$ DTT for $30 \mathrm{~min}$ at $37^{\circ} \mathrm{C}$. Fluorescence was detected in the Fluoroskan AscentTM equipment (Thermo Labsystems, Egelsbach, Germany), and the relative fluorescence units (RFU) were normalized to the protein concentration.

Constructs, transfection, retroviral and lentiviral infections. Lentiviral shRNA to knock down human Bax, Bak, Bad and mouse Bim was obtained from Sigma. As a control, a scrambled shRNA was used (Ctrl, SHC002). To produce lentiviruses, $70-80 \%$ confluent HEK293T cells were transfected with a combination of $1.5 \mu \mathrm{g}$ of pMD2.G encoding the viral envelope, $3 \mu \mathrm{g}$ of the pSPAX2 packaging plasmid and $5 \mu \mathrm{g}$ of the lentiviral vector encoding the shRNA of interest, using the SuperFect transfection reagent (Qiagen, Hilden, Germany). After 3 days of culturing in $5 \mathrm{mM}$ sodium butyrate the lentivirus-containing medium was removed, passed through a $0.45 \mu \mathrm{m}$ filter, supplemented with $5 \mu \mathrm{g} / \mathrm{ml}$ polybrene and used to infect cells. The CDNAs of WT and mutant Bim $E L$ were re-introduced into Bax/Bak DKO and Bim KO MEFs by retroviral transduction. As retroviral vector, the pMIG containing an IRES-controlled GFP cassette for co-expression and a puromycinresistance gene was used. Three micrograms of the PMIG vector was mixed with $3 \mu \mathrm{g}$ of the pVSVG envelope vector and $3 \mu \mathrm{g}$ of the packaging vector HIT60 and transfected into HEK293T cells to produce retroviruses as described above for lentiviruses. A number of $2 \times 10^{5}$ cells of BEAS-2B or MEF cells were grown in a well of a six-well plate, treated with $400 \mu \mathrm{l}$ of the retroviral or lentiviral supernatants and centrifuged at 2000 r.p.m. at room temperature for $10 \mathrm{~min}$. After $1-3 \mathrm{~h}$ of infection, the cells were washed with PBS, cultured in full media for at least $18 \mathrm{~h}$ and then either selected with puromycin for stable expression of the gene of interest or FACS sorted for the cells co-expressing GFP. The T38A, S100A, S112A, S114A single, T112A/S114A double and S100A/T112A/S114A triple mutations were

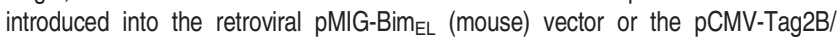
FLAG-Bim $_{E L}$ plasmid by using the Quickchange Site-directed Mutagenesis kit (Agilent, Böblingen, Germany) (primers in Supplementary Table S1) according to the protocol recommended by the manufacturer. All constructs were confirmed by sequencing.

In vivo phosphorylation and anti-FLAG and anti-Bak immunoprecipitations. A number of $2 \times 10^{6}$ HEK 293T cells were transiently transfected with $\mathrm{pCMV-Tag2B}$ vectors expressing FLAG-tagged WT Bim $\mathrm{EL}_{\mathrm{L}}$ or its T112A, T112A/S114A or S100A/T112A/S114A phosphodeficient mutants, using the SuperFect reagent as described by the manufacturer (Qiagen). After $24 \mathrm{~h}$, cells were washed in PBS and incubated in phosphate-free medium for $30 \mathrm{~min}$ to remove most of the unlabeled phosphate. Subsequently, $50 \mu \mathrm{Ci} / \mathrm{ml}$ ${ }^{33} \mathrm{P}$-orthophosphate $(1 \mathrm{mCi}=37 \mathrm{mBq})$ was added to the cells in phosphatefree medium for $30 \mathrm{~min}$, and the cells were treated (or not) with $1 \mu \mathrm{M}$ GT for $6 \mathrm{~h}$. The cells were lysed in whole-cell lysis buffer (buffer A $+1 \%$ CHAPS) and directly subjected to anti-FLAG immunoprecipitation (IP) using the Sigma EZview Red Anti-FLAG M2 Affinity beads. For each pull-down, $40 \mu$ l of beads were aliquoted and equilibrated with $500 \mu \mathrm{l}$ of whole-cell lysis buffer (containing all inhibitors). Beads were always centrifuged at $8200 \times g$ for $30 \mathrm{~s}$ at $4{ }^{\circ} \mathrm{C}$. Subsequently, the supernatant was discarded and $500 \mu \mathrm{l}$ of the ${ }^{33} \mathrm{P}$-labeled whole-cell lysates were mixed with the beads and incubated on the shaker for $2 \mathrm{~h}$ at $4{ }^{\circ} \mathrm{C}$. After the beads were spun down, the supernatant was discarded and kept for eventual analysis. The beads were washed three times with whole-cell lysis buffer, and finally the FLAG-tagged protein was eluted/released from the beads with $50 \mu \mathrm{l} 3 \times \mathrm{FLAG}$ peptide. The eluted sample was run on SDS-PAGE and subjected to either antipT112 western blot analysis to detect phosphorylation at T112 of Bim $\mathrm{EL}_{\mathrm{L}}$ or to autoradiography to detect ${ }^{33} \mathrm{P}$ labeling of $\mathrm{Bim}_{\mathrm{EL}}$. To detect FLAG-Bim $\mathrm{EL}_{\mathrm{EL}} / \mathrm{BCl}-2$ and FLAG-Bim $\mathrm{EL}_{\mathrm{EL}} / \mathrm{BCl}-\mathrm{X}_{\mathrm{L}}$ interactions, unlabeled whole-cell lysates were immunoprecipitated with anti-FLAG antibodies and eluted by $3 \times$ FLAG peptide as described above. The eluates were then subjected to anti-Bim, anti-pT112 Bim, anti-Bcl-2 or anti-Bcl-x immunoblotting. To determine the conformational change of Bak (which reflects its active state), $500 \mu$ l of unlabeled whole-cell lysates were subjected to immunoprecipitation using $3 \mu \mathrm{g}$ of the mouse monoclonal anti-Bak Ab-1 antibody, which detects the N-terminus of Bak only when it is in an open, active state. After washing in whole-cell lysis buffer, the immunoprecipitates were directly solubilized in Lämmli buffer and subjected to anti-Bak immunoblotting (rabbit polyclonal, Upstate, \#06-536).
Mitochondria isolation and in vitro cytochrome $c$ release. Mitochondria were isolated from 20 confluent $150 \mathrm{~mm}$ culture dishes of BEAS-2B cells. The cells were collected by trypsinization, centrifuged, resuspended in $500 \mu \mathrm{l}$ ice-cold AT-buffer ( $300 \mathrm{mM}$ trehalose, $10 \mathrm{mM}$ HEPES-KOH, pH 7.5, $80 \mathrm{mM} \mathrm{KCl}$, $1 \mathrm{mM}$ EGTA, $1 \mathrm{mM}$ EDTA, $0.1 \%$ BSA and $25 \times$ protease inhibitor cocktail) and incubated on ice for $15 \mathrm{~min}$ to swell. The cells were lysed by passing them through a 23G needle 35-40 times. The suspension was cleared off insoluble debris and spun down at $13000 \times g$ for $10 \mathrm{~min}$. The crude mitochondrial pellet was resuspended in $50 \mu \mathrm{l}$ SEM buffer $(250 \mathrm{mM}$ sucrose, $10 \mathrm{mM}$ HEPES, pH 7.2, $140 \mathrm{mM} \mathrm{KCl}$, including protease inhibitors) and used for the in vitro cytochrome $c$ release assay. The AT buffer had to be replaced by SEM buffer because otherwise cytochrome $c$ release would have been difficult (trehalose extensively stabilizes mitochondria). Equal amounts of crude mitochondria (60-100 $\mu$ g protein) were incubated for $2 \mathrm{~h}$ at $37^{\circ} \mathrm{C}$ with 0,20 and $50 \mu \mathrm{M}$ of normal GT (oxidized, oxGT) or GT previously reduced with $2 \mathrm{mM}$ glutathione at $30^{\circ} \mathrm{C}$ for $60 \mathrm{~min}$ (reduced, redGT). As a positive control for cytochrome $c$ release, $20 \mathrm{nM}$ recombinant His-Bax and/or $5 \mathrm{nM}$ recombinant tBid (both kindly provided by Jean-Claude Martinou) were added to the incubation mix. The mitochondria were then separated from the supernatant by centrifugation at $13000 \times g$ for $10 \mathrm{~min}$ at $4{ }^{\circ} \mathrm{C}$, and both fractions were separately analyzed by immunoblotting using a mouse monoclonal anti-cytochrome $c$ antibody $(1: 1000, \mathrm{BD}$ Pharmingen, Heidelberg, Germany).

RNA extraction and RT-qPCR. Total RNA was isolated from non-treated and GT-treated ( $\pm 20 \mu \mathrm{M}$ of the PI3K inhibitor LY294002) MEFs (for Bim) or untreated BEAS-2B cells (for p38 MAPK isoforms) by the Qiagen RNA easy Mini Kit according to the manufacturer's protocol (Qiagen). Five micrograms of total RNA was reverse transcribed using the Invitrogen Superscript First Strand Synthesis Kit (Invitrogen, Karlsruhe, Germany) and the therein provided random hexamer primers. The quantitative PCR ( (qPCR) was performed in 96-well plates using $12.5 \mu \mathrm{l}$ Mesa Blue qPCR Mix (including Hotstar meteor Taq polymerase), $0.5 \mu \mathrm{l}$ of each forward and reverse primer for the candidate gene (10 $\mu \mathrm{M}$ stock), $9.5 \mu \mathrm{l}$ of distilled $\mathrm{H}_{2} \mathrm{O}$ and $2 \mu \mathrm{l}$ of the reverse-transcribed cDNA per well. As a negative control, $\mathrm{H}_{2} \mathrm{O}$ was used instead of the master mix and for normalization the housekeeping gene $L 32$ was co-amplified. The plate was sealed with adhesive foil, spun down at 1000 r.p.m. for $1 \mathrm{~min}$ and then subjected to the PCR reaction in a Biorad MyiQ Real-time PCR machine (Biorad, Munich, Germany). For each cDNA sample, triplicates were measured with the two primer pairs (gene of interest and housekeeping gene). The primers are given in Supplementary Table S1.

Cultivation of fungi. Wild-type, GT-deficient ( $\Delta$ GliP) and GT-reconstituted (GliR) A. fumigatus strains were kindly obtained from Janyce A. Sugui. ${ }^{36}$ They were either cultivated as monolayer biofilm on solid medium or as filamentous fungus in liquid at $37^{\circ} \mathrm{C}$ without $\mathrm{CO}_{2}$. Conidia/spores were produced on solid SABOURAUD agar plates. After the fungus had overgrown the plate (ca. $48 \mathrm{~h}$ ), the conidia were rinsed off with $10 \mathrm{ml}$ PBS/Tween $0.05 \%$ and counted in a Neubauer chamber. To obtain a fungal supernatant containing optimal concentrations of GT for cellular studies, $1 \times 10^{7}$ A. fumigatus. conidia were seeded in $50 \mathrm{ml} \mathrm{RPMI} / 25 \mathrm{mM}$ HEPES medium. Fungal media was collected after $72 \mathrm{~h}$, at which time point the fungus had to be grown filamentous for an efficient secretion of the GT. Before using the fungal supernatant for cell culture experiments, it was sterile filtered to get rid of any fungal hyphae and conidia. All experiments with $A$. fumigatus. were handled in accordance with the German law for infection protection.

Statistics. Statistical significance was analyzed by a two-tailed Student's t-test. Data are the means of at least three experiments \pm S.E.M.

\section{Conflict of Interest}

The authors declare no conflict of interest.

Acknowledgements. We thank Georg Häcker, Freiburg, for the pMIG-BimeL plasmid and the FLAG-Bim ${ }_{E L}$ CDNA, Andreas Strasser, WEHI, Melbourne, for Bim KO, other BH3-only KO and Bax/Bak DKO MEFs, Jean-Claude Martinou, Geneva, for recombinant tBid and Bax and Janyce A. Sugui, NIH Bethesda, for the WT and mutant Aspergillus fumigatus strains. We thank Tilman Brummer, Freiburg, for his advice and encouragements. This work was supported by the Centre of Chronic Immunodeficiency $(\mathrm{CCl})$ funded by the BMBF, Germany (to $A G$ and $\mathrm{CB}$ ), the Spemann Graduate School of Biology and Medicine (SGBM, GSC-4) funded by the 
Excellence Initiative of the German Federal and State Governments, Germany (to AG, FH and CB), by the Centre for Biological Signalling Studies (BIOSS, EXC-294 funded by the Excellence Initiative, Germany (to $\mathrm{CB}$ and $\mathrm{UM}$ ) and by the Deutsche Forschungsgemeinschaft (DFG ID7/4-2, to MI). RJD is an investigator of the Howard Hughes Medical Institute.

\section{Author contributions}

$\mathrm{CB}, \mathrm{UM}$ and $\mathrm{MMS}$ conceived the project. $\mathrm{AG}, \mathrm{FH}, \mathrm{DOF}, \mathrm{KW}$ carried out the experiments. RJD contributed the knockout/in mouse lines and the pT112 Bim antibody. MI contributed the primary alveolar epithelial type II cells. CB, AG and FH prepared the figures and wrote the manuscript.

1. Latge JP. The pathobiology of Aspergillus fumigatus. Trends Microbiol 2001; 9: 382-389.

2. Romani L. Immunity to fungal infections. Nat Rev Immunol 2004; 4: 1-13.

3. Segal BH, Walsh TJ. Current approaches to diagnosis and treatment of invasive aspergillosis. Am J Respir Crit Care Med 2006; 173: 707-717.

4. Kupfahl C, Michalka A, Lass-Flörl C, Fischer G, Haase G, Ruppert T et al. Gliotoxin production by clinical and environmental Aspergillus fumigatus strains. Int J Med Microbiol 2007; 298: 319-327.

5. Müllbacher A, Waring $P$, Tiwari-Palni U, Eichner RD. Structural relationship of epipolythiodioxopiperazines and their immunomodulating activity. Mol Immunol 1986; 23: 231-235.

6. Eichner RD, Waring P, Geue AM, Braithwaite AW, Müllbacher A. Gliotoxin causes oxidative damage to plasmid and cellular DNA. J Biol Chem 1988; 263: 3772-3777.

7. Pahl HL, Krauss B, Schulze-Osthoff K, Decker T, Traenckner EB, Vogt $M$ et al. The immunosuppressive fungal metabolite gliotoxin specifically inhibits transcription factor NF- kappaB. J Exp Med 1996; 183: 1829-1840.

8. Suen YK, Fung KP, Lee CY, Kong SK. Gliotoxin induces apoptosis in cultured macrophages via production of reactive oxygen species and cytochrome $c$ release without mitochondrial depolarization. Free Radic Res 2001; 35: 1-10.

9. Kweon YO, Paik YH, Schnabl B, Qian T, Lemasters JJ, Brenner DA. Gliotoxin-mediated apoptosis of activated human hepatic stellate cells. J Hepatol 2003; 39: 38-46.

10. Pardo J, Urban C, Galvez EM, Ekert EG, Müller U, Kwon-Chung J et al. The mitochondria protein Bak is pivotal for gliotoxin-induced apoptosis and a critical host factor of Aspergillus fumigatus virulence in mice. J Cell Biol 2006; 174: 509-519.

11. Chipuk JE, Moldoveanu T, Llambi F, Parsons MJ, Green DR. The Bcl-2 family reunion. Mol Cell 2010; 37: 299-310.

12. Riedl SJ, Salvesen GS. The apoptosome: signaling platform of cell death. Nat Rev Mol Cell Biol 2007; 8: 405-413.

13. Pinon JD, Labi V, Egle A, Villunger A. Bim and Bmf in tissue homeostasis and malignant disease. Oncogene 2008; 27: S41-S52.

14. O'Connor L, Strasser A, O'Reilly LA, Hausmann G, Adams JM, Cory S et al. Bim: a novel member of the Bcl-2 family that promotes apoptosis. EMBO J 1998; 17: 384-395.

15. Dijkers PF, Medema RH, Lammers JW, Koenderman L, Coffer PJ. Expression of the proapoptotic Bcl-2 family member Bim is regulated by the forkhead transcription factor FKHR L1. Curr Biol 2000; 10: 1201-1204.

16. Puthalakath $\mathrm{H}$, Huang DCS, O'Reilly LA, King SM, Strasser A. The pro-apoptotic activity of the $\mathrm{Bcl}-2$ family member Bim is regulated by interaction with the dynein motor complex. Mol Cell 1999; 3: 287-296.
17. Luciano F, Jacquel A, Colosetti P, Herrant M, Cagnol S, Pages G et al. Phosphorylation of Bim-EL by Erk $1 / 2$ on serine 69 promotes its degradation via the proteasome pathway and regulates ist proapoptotic function. Oncogene 2003; 22: 6785-6793.

18. Ley R, Ewings KE, Hadfield K, Cook SJ. Regulatory phosphorylation of Bim: sorting out the ERK from the JNK. Cell Death Differ 2005; 12: 1008-1014.

19. Clybouw C, Merino D, Nebl T, Masson F, Robati M, O'Reilly A et al. Alternative splicing of Bim and Erk-mediated Bim(EL) phosphorylation are dispensable for hematopoietic homeostasis in vivo. Cell Death Differ 2012; 19: 1060-1068.

20. Hübner A, Barrett T, Flavell RA, Davis RJ. Multisite phosphorylation regulates Bim stability and apoptotic activity. Mol Cell 2008; 30: 415-425.

21. Lei K, Davis RJ. JNK phosphorylation of Bim-related members of the Bcl2 family induces Bax-dependent apoptosis. Proc Natl Acad Sci USA 2003; 100: 2432-2437.

22. Corazza N, Jakob S, Schaer C, Frese S, Keogh A, Stroka D et al. TRAlL receptormediated JNK activation and Bim phosphorylation critically regulate Fas-mediated liver damage and lethality. J Clin Invest 2006; 116: 2493-2499.

23. Waring $\mathrm{P}$, Newcombe $\mathrm{N}$, Edel $\mathrm{M}$, Lin $\mathrm{QH}$, Jiang $\mathrm{H}$, Sjaarda $\mathrm{A}$ et al. Cellular uptake and release of the immunomodulating fungal toxin gliotoxin. Toxicon 1994; 32 : 491-504.

24. Kuwana T, Mackey MR, Perkins G, Ellisman MH, Latterich M, Schneiter R et al. Bid, Bax, and lipids cooperate to form supramolecular openings in the outer mitochondrial membrane. Cell 2002; 111: 331-342.

25. Gilmore AP. Anoikis. Cell Death Differ 2005; 12(Suppl 2): 1473-1477.

26. Seo M, Lee YI, Cho CH, Bae CD, Kim IH, Juhnn YS et al. Bi-directional regulation of UV- induced activation of $\mathrm{p} 38$ kinase and $\mathrm{c}$-Jun $\mathrm{N}$-terminal kinase by $\mathrm{G}$ protein beta gamma- subunits. J Biol Chem 2002; 277: 24197-24203.

27. Steelman LS, Abrams SL, Whelan J, Bertrand FE, Ludwig DE, Bäsecke J et al. Contributions oft he Raf/MEK/ERK, PI3K/PTEN/Akt/mTOR and Jak/STAT pathways to leukemia. Leukemia 2008; 22: 686-707.

28. Zha J, Harada E, Yang E, Jockel J, Korsmeyer SJ. Serine phosphorylation of death agonist BAD in response to survival factor results in binding to 14-3-3 not BCL-X(L). Cell 1996; 87: 619-628

29. Reginato MJ, Mills KR, Paulus JK, Lynch DK, Sgroi DC, Debnath J et al. Integrins and EGFR coordinately regulate the pro-apoptotic protein Bim to prevent anoikis. Nat Cell Biol 2003; 5: 733-740.

30. Wang P, Gilmore AP, Streuli $\mathrm{CH}$. Bim is an apoptosis sensor that responds to loss of survival signals delivered by epidermal growth factor but not those provided by integrins. J Biol Chem 2004; 279: 41280-41285.

31. Hall A. Rho GTPases and the control of cell behaviour. Biochem Soc Trans 2005; 33 891-895.

32. Meng Q, Xia Y. c-Jun, at the crossroad of the signaling network. Protein Cell 2001; 2: $889-898$

33. Xu Z, Kukekov NV, Greene LA. POSH acts as a scaffold for a multiprotein complex that mediates JNK activation in apoptosis. EMBO J 2003; 22: 252-261.

34. Corti $\mathrm{M}$, Brody $\mathrm{AF}$, Harrison $\mathrm{JH}$. Isolation and primary culture of muring alveolar type II cells. Am J Respir Cell Mol Biol 1996; 14: 309-315.

35. Vogel S, Raulf N, Bregenhorn S, Biniossek M, Maurer U, Czabotar P et al. Cytosolic Bax: does it require binding proteins to keep its pro-apoptotic activity in check? J Biol Chem 2012; 287: 9112-9127.

36. Sugui JA, Pardo J, Chang YC, Zarember KA, Nardone G, Galvez A et al. Gliotoxin is a virulence factor of Aspergillus fumigatus: gliP deletion attenuates virulence in mice immunosuppressed with hydrocortisone. Eukaryot Cell 2007; 6: 1562-1569.

Supplementary Information accompanies this paper on Cell Death and Differentiation website (http://www.nature.com/cdd) 